\title{
Characterizing the absolute continuity of the convolution of orbital measures in a classical Lie algebra
}

\author{
Sanjiv Kumar Gupta and Kathryn E. Hare
}

\begin{abstract}
Let $\mathfrak{g}$ be a compact, simple Lie algebra of dimension $d$. It is a classical result that the convolution of any $d$ non-trivial, $G$-invariant, orbital measures is absolutely continuous with respect to Lebesgue measure on $\mathfrak{g}$ and the sum of any $d$ non-trivial orbits has non-empty interior. The number $d$ was later reduced to the rank of the Lie algebra (or rank +1 in the case of type $A_{n}$ ). More recently, the minimal integer $k=k(X)$ such that the $k$-fold convolution of the orbital measure supported on the orbit generated by $X$ is an absolutely continuous measure was calculated for each $X \in \mathfrak{g}$.

In this paper $\mathfrak{g}$ is any of the classical, compact, simple Lie algebras. We characterize the tuples $\left(X_{1}, \ldots, X_{L}\right)$, with $X_{i} \in \mathfrak{g}$, which have the property that the convolution of the $L$-orbital measures supported on the orbits generated by the $X_{i}$ is absolutely continuous and, equivalently, the sum of their orbits has non-empty interior. The characterization depends on the Lie type of $\mathfrak{g}$ and the structure of the annihilating roots of the $X_{i}$. Such a characterization was previously known only for type $A_{n}$.
\end{abstract}

\section{Introduction}

Let $G$ be a compact, connected simple Lie group and $\mathfrak{g}$ its Lie algebra. Given $X \in \mathfrak{g}$, we let $\mu_{X}$ denote the $G$-invariant, orbital measure supported on $O_{X}$, the orbit generated by $X$ under the adjoint action of $G$. Geometric properties of the Lie algebra ensure that if a suitable number of non-trivial orbits are added together the resulting subset of $\mathfrak{g}$ has non-empty interior and if a suitable number of orbital measures are convolved together, the resulting measure is absolutely continuous with respect to the Lebesgue measure on $\mathfrak{g}$. From the work of Ragozin in [18 it can be seen that the dimension of the Lie algebra is a 'suitable number'.

In a series of papers (see [9] and [10 and the papers cited therein) the authors, with various coauthors, improved upon Ragozin's result determining, for each $X \in$ $\mathfrak{g}$, the integer $k(X)$ with the property that $\mu_{X}^{k}$ is absolutely continuous for all $k \geq k(X)$ and $\mu_{X}^{k}$ is singular to Lebesgue measure otherwise (where $\mu_{X}^{k}$ denotes the

1991 Mathematics Subject Classification. Primary 43A80; Secondary 17B45, 58C35.

Key words and phrases. compact Lie algebra, orbital measure, absolutely continuous measure.

The first author would like to thank the Dept. of Pure Mathematics at the University of Waterloo and the second author the School of Mathematics and Statistics at St. Andrews University for their hospitality while some of this research was done. This research was supported in part by the Edinburgh Math. Society, NSERC and Sultan Qaboos University. 
$k$-fold convolution). Furthermore, the $k$-fold sum of $O_{X}$ has non-empty interior if $k \geq k(X)$ and otherwise has measure zero. A formula was given for $k(X)$ depending on combinatorial properties of the annihilating roots of $X$. In particular, it was shown that the convolution of any $r$ orbital measures is absolutely continuous if and only if $r$ is at least the rank of the Lie algebras when $\mathfrak{g}$ is of type $B_{n}, C_{n}$ or $D_{n}$ and $r$ is at least rank+1 for the Lie algebras of type $A_{n}$. The proofs relied heavily upon representation theory and harmonic analysis.

By taking a geometric approach, Wright in [23] extended these results in the special case of the classical Lie algebra $\mathfrak{g}=s u(n)$ (type $A_{n-1}$ ), proving that $\mu_{X_{1}} *$ $\cdots * \mu_{X_{L}}$ is absolutely continuous with respect to Lebesgue measure if and only if $\sum_{i=1}^{L} s_{i} \geq n(L-1)$ where $s_{i}$ is the dimension of the largest eigenspace of the $n \times n$ matrix $X_{i}$, provided it is not the case that $L=2, n \geq 4$ is even, and $X_{1}, X_{2}$ each have two distinct eigenvalues, both of multiplicity $n / 2$.

Using primarily algebraic methods, Gracyzk and Sawyer (c.f., 4, 5]), addressed analogous problems in the setting of a non-compact, symmetric space, improving upon other work of Ragozin, 17. In particular, they characterized when the convolution of two (possibly different) bi-invariant measures is absolutely continuous in the symmetric spaces $s l(n, F) / s u(n, F)$ (where the restricted root system is also type $\left.A_{n-1}\right)$.

Inspired by their methods, in this paper we characterize the $L$-tuples, $\left(X_{1}, \ldots, X_{L}\right)$ with $X_{i} \in \mathfrak{g}$, such that the convolution $\mu_{X_{1}} * \cdots * \mu_{X_{L}}$ is absolutely continuous when the Lie algebra is any one of the classical Lie algebras (those of type $A_{n}, B_{n}, C_{n}$ or $D_{n}$ ), leaving only one pair in $D_{n}$ where we have been unable to decide the answer. As well, this characterizes the $L$-tuples such that $\sum_{i=1}^{L} O_{X_{i}}$ has non-empty interior in $\mathfrak{g}$ as opposed to measure zero. As Wright found with type $A_{n}$, the characterization can be expressed most simply as a function of the dimensions of the largest eigenspaces of the $X_{i}$ when these are viewed as matrices in the classical matrix Lie algebras (see Section 3 for the precise statement). The characterization can also be described in terms of the root structure of the set of annihilating roots of the $X_{i}$, as was done in the previous study of convolutions of a single orbital measure. Our argument is completely different from that used by Wright and from the harmonic analysis - representation theory approach used by the authors previously. It relies heavily upon the (algebraic) Lie theory of roots and root vectors.

Using these results, we also obtain a similar characterization of the absolute continuity of the convolution products of $G$-invariant measures, $\mu_{x_{i}}$, supported on conjugacy classes $C_{x_{i}}$ in $G$, for the elements $x_{i} \in G$ whose annihilating roots agree with those of a preimage of $x_{i}$ in $\mathfrak{g}$ under the exponential map. This extends work of [8] where the minimal integer $k(x)$ with the property that $\mu_{x}^{k(x)}$ is absolutely continuous was determined.

In a future paper, we will adapt our general strategy to improve upon Gracyzk and Sawyer's symmetric space results.

Finding the density function, or Radon Nikodym derivative, of the absolutely continuous measure $\mu_{X_{1}} * \cdots * \mu_{X_{L}}$ is a challenging problem. In the case of the convolution of two orbital measures in $s u(n)$, this has been computed in [2]. A general formula for the convolution of two orbital measures in terms of the projection of such measures to maximal tori was found in [1. The density function for the analogous problem on non-compact symmetric spaces was studied in [3] (and see also the references cited there). In [15, the sum of two adjoint orbits in $s u(n)$ 
is explicitly described in terms of a system of linear equations, but for more than 2 -fold sums this too seems very difficult. Other work investigating the smoothness properties of convolutions of measures supported on manifolds whose product has non-empty interior was carried out by Ricci and Stein in [19] and [20.

The paper is organized as follows: In section 2 we review background material in Lie theory and introduce basic notation. In section 3 we state the main result. The necessity of our characterization is proven in section 4 . In section 5 we establish the general strategy for tackling the absolute continuity problem and then complete the proof of the main theorem in section 6 . In section 7 we discuss consequences of our result and deduce the absolute continuity result for convolutions of orbital measures on Lie groups mentioned above.

\section{Notation and Background}

2.1. Notation. We begin by establishing notation and reviewing basic facts about roots and root vectors. Assume $G_{n}$ is a classical, compact, connected simple Lie group of rank $n$, one of type $A_{n}, B_{n}, C_{n}$ or $D_{n}$. We denote by $\mathfrak{g}_{n}$ its (real) Lie algebra, $\mathfrak{t}_{n}$ a maximal torus of $\mathfrak{g}_{n}$ and $W$ the Weyl group.

We write $[\cdot, \cdot]$ for the Lie bracket action. The map ad $: \mathfrak{g}_{n} \rightarrow \mathfrak{g}_{n}$ is given by $\operatorname{ad}(X)(Y)=[X, Y]$. The exponential function, exp, is a surjection of $\mathfrak{g}_{n}$ onto $G_{n}$, and $G_{n}$ acts on $\mathfrak{g}_{n}$ by the adjoint action, denoted $\operatorname{Ad}(\cdot)$. Recall that for $M \in \mathfrak{g}_{n}$,

$$
A d(\exp M)=\exp (a d(M))=I d+\sum_{k=1}^{\infty} \frac{a d^{k}(M)}{k !}
$$

where $a d^{k}(M)$ is the $k$-fold composition of $a d(M)$.

By an orbit of an element $X \in \mathfrak{g}_{n}$, we mean the subset

$$
O_{X}:=\left\{\operatorname{Ad}(g)(X): g \in G_{n}\right\} \subseteq \mathfrak{g}_{n} .
$$

There is no loss in assuming $X$ belongs to $\mathfrak{t}_{n}$ since every orbit contains a torus element. Orbits are compact manifolds of proper dimension in $\mathfrak{g}_{n}$ and hence of Lebesgue measure zero. If $X=0$, then $O_{X}=\{0\}$ is a singleton, but otherwise $O_{X}$ has positive dimension.

By the orbital measure, $\mu_{X}$, we mean the probability measure invariant under the adjoint action of $G_{n}$ and compactly supported on $O_{X}$. It integrates bounded, continuous functions $f$ on $\mathfrak{g}_{n}$ by the rule

$$
\int_{\mathfrak{g}_{n}} f d \mu_{X}=\int_{G_{n}} f(A d(g) X) d g
$$

where $d g$ is the Haar measure on $G_{n}$. The orbital measures are singular to Lebesgue measure since their supports have Lebesgue measure zero. Except in the special case when $X=0, \mu_{X}$ is an example of a continuous measure, meaning the $\mu_{X}$ measure of any singleton is zero.

The classical Lie groups and algebras are said to be of type $A_{n}$ for $n \geq 1, B_{n}$ for $n \geq 2, C_{n}$ for $n \geq 3$ or $D_{n}$ for $n \geq 4$. This means that the root system of the complexified Lie algebra with respect to the complexified torus, denoted $\Phi_{n}$, is of that Lie type. It is often convenient to refer to type $A_{n}$ as type $S U(n+1)$ for reasons that will become clear later.

For the convenience of the reader we describe $\Phi_{n}$ below for each of the classical types. Note that by $e_{j}$ we mean the $j^{\prime}$ th standard basis vector of $\mathbb{R}^{n}$ (or in $\mathbb{R}^{n+1}$ in the case of type $A_{n}$ ). The real span of $\Phi_{n}$, denoted $s p \Phi_{n}$, is equal to $\mathbb{R}^{n}$ (or the 
subspace of $\mathbb{R}^{n+1}$ spanned by the standard vectors $e_{j}-e_{n+1}$ for $j=1, \ldots, n$ in the case of type $A_{n}$ ).

\begin{tabular}{|cc|}
\hline Lie algebra & Root system $\Phi_{n}$ \\
$A_{n}$ & $\left\{ \pm\left(e_{i}-e_{j}\right): 1 \leq i<j \leq n+1\right\}$ \\
$B_{n}$ & $\left\{ \pm e_{i}, \pm e_{i} \pm e_{j}: 1 \leq i \neq j \leq n\right\}$ \\
$C_{n}$ & $\left\{ \pm 2 e_{i}, \pm e_{i} \pm e_{j}: 1 \leq i \neq j \leq n\right\}$ \\
$D_{n}$ & $\left\{ \pm e_{i} \pm e_{j}: 1 \leq i \neq j \leq n\right\}$ \\
\hline
\end{tabular}

In the case of type $A_{n}$, the Weyl group is the group of permutations on the letters $\{1, \ldots, n+1\}$. For types $B_{n}, C_{n}$ (and $D_{n}$ ), the Weyl groups are the group of permutations on $\{1, \ldots, n\}$, together with (an even number of) sign changes.

These Lie algebras and groups can be identified with the classical matrix algebras and groups listed below. All compact, connected simple Lie groups are homomorphic images by finite subgroups of these classical matrix groups.

- $s u(n)$ - the set of $n \times n$ skew-Hermitian, trace zero matrices is the model we use for the Lie algebra of type $A_{n-1}$. $S U(n)$ - the $n \times n$ special unitary matrices is a compact Lie group of type $A_{n-1}$.

- $s o(p)$ - the set of $p \times p$ real, skew-symmetric matrices. When $p=2 n$ it is the Lie algebra of type $D_{n}$ and when $p=2 n+1$ it is of type $B_{n} . S O(p)$ the $p \times p$ special orthogonal matrices are associated compact Lie groups.

- $s p(n)$ - the set of $2 n \times 2 n$ matrices of the form $\left[\begin{array}{cc}A & \frac{B}{B} \\ -\bar{A}\end{array}\right]$ where $A, B$ are complex $n \times n$ matrices with $B$ symmetric and $A$ skew-Hermitian is the Lie algebra of type $C_{n}$. The $n$ 'th order symplectic group, $S p(n)$, is the set of $2 n \times 2 n$ unitary matrices $U$ satisfying $U^{t r} J U=J$, where $J=\left[\begin{array}{cc}0 & -I \\ I & 0\end{array}\right]$ with $I$ being the $n \times n$ identity matrix. $S p(n)$ is a compact Lie group of type $C_{n}$.

For each root $\alpha \in \Phi_{n}$, we let $E_{\alpha}$ denote a corresponding root vector so that if $H \in \mathfrak{t}_{n}$, then

$$
\left[H, E_{\alpha}\right]=i \alpha(H) E_{\alpha} .
$$

(We make the convention that roots are real valued.) We will choose a collection of root vectors, $\left\{E_{\alpha}\right\}$, that form a Weyl basis (see [21, p. 290]). In particular, this ensures that if $\alpha, \beta$ and $\alpha+\beta$ are roots, then there are non-zero scalars $N_{\alpha, \beta}$ satisfying $N_{\alpha, \beta}=N_{-\alpha,-\beta}$ and

$$
\left[E_{\alpha}, E_{\beta}\right]=N_{\alpha, \beta} E_{\alpha+\beta} .
$$

If $\alpha+\beta$ is not a root, then $\left[E_{\alpha}, E_{\beta}\right]=0$.

The root vector, $E_{\alpha}$, can be written in a unique way as $E_{\alpha}=R E_{\alpha}+i I E_{\alpha}$, where $R E_{\alpha}$ and $I E_{\alpha}$ both belong to the (real) Lie algebra $\mathfrak{g}_{n}$. We refer to these as the real and imaginary parts of the root vector. We write $F E_{\alpha}$ if we mean either $R E_{\alpha}$ or $I E_{\alpha}$. One can easily see that $E_{-\alpha}=R E_{\alpha}-i I E_{\alpha}$. Furthermore, $R E_{\alpha}=\left(E_{\alpha}+E_{-\alpha}\right) / 2$ and $I E_{\alpha}=\left(E_{\alpha}-E_{-\alpha}\right) /(2 i)$.

The vector space spanned by $R E_{\alpha}$ and $I E_{\alpha}$ over various sets of roots $\alpha$ will be important to us. In particular, we put

$$
\mathcal{V}_{n}=\left\{R E_{\alpha}, I E_{\alpha}: \alpha \in \Phi_{n}^{+}\right\} \subseteq \mathfrak{g}_{n}
$$


where $\Phi_{n}^{+}$denotes the subset of positive roots. With this notation the Lie algebra can be decomposed as

$$
\mathfrak{g}_{n}=\mathfrak{t}_{n} \bigoplus_{\alpha \in \Phi_{n}^{+}} s p\left\{R E_{\alpha}, I E_{\alpha}\right\}=\mathfrak{t}_{n} \bigoplus s p \mathcal{V}_{n}
$$

where $s p$ denotes the real span. Thus the dimension of $\mathfrak{g}_{n}$ is equal to $n+\left|\Phi_{n}\right|$.

From (2.1) it follows that

$$
\left[H, R E_{\alpha}\right]=-\alpha(H) I E_{\alpha} \text { and }\left[H, I E_{\alpha}\right]=\alpha(H) R E_{\alpha} .
$$

It is also well known that

$$
\left[R E_{\alpha}, I E_{\alpha}\right]=\frac{-1}{2 i}\left[E_{\alpha}, E_{-\alpha}\right]
$$

is a non-zero element of the maximal torus. It should be noted that if $\left\{\alpha_{j}: j \in\right.$ $J\} \subseteq \Phi_{n}$ is a spanning set for $s p \Phi_{n}$, then $\left.\left\{R E_{\alpha_{j}}, I E_{\alpha_{j}}\right]: j \in J\right\}$ spans $\mathfrak{t}_{n}$.

Since $\left\{E_{\alpha}\right\}$ is a Weyl basis, we have

$$
\begin{aligned}
{\left[R E_{\alpha}, R E_{\beta}\right] } & =c R E_{\alpha+\beta}+d R E_{\beta-\alpha}, \\
{\left[R E_{\alpha}, I E_{\beta}\right] } & =c I E_{\alpha+\beta}+d I E_{\beta-\alpha} \\
{\left[I E_{\alpha}, I E_{\beta}\right] } & =-c R E_{\alpha+\beta}+d R E_{\beta-\alpha},
\end{aligned}
$$

where $R E_{\gamma}$ and $I E_{\gamma}$ should be understood to be the zero vector if $\gamma$ is not a root and $c=N_{\alpha, \beta} / 2, d=N_{\alpha,-\beta} / 2$.

We refer the reader to [12, 14] and [21 for proofs of these well known facts and further details on the representation theory of Lie algebras.

2.2. Annihilating roots. We call a root, $\alpha$, an annihilating root of $X \in t_{n}$ if $\alpha(X)=0$ and call $\alpha$ a non-annihilating root of $X$ otherwise. The set of annihilating roots of $X$,

$$
\Phi_{X}:=\{\alpha \in \Phi: \alpha(X)=0\},
$$

is a root subsystem of $\Phi_{n}$. As we will see, these root subsystems are critical for understanding properties about orbits and orbital measures, as are the associated root vectors. We will denote by

$$
\mathcal{N}_{X}:=\left\{R E_{\alpha}, I E_{\alpha}: \alpha \notin \Phi_{X}\right\} \subseteq \mathcal{V}_{n},
$$

the linearly independent subset of $\mathcal{V}_{n}$ consisting of the real and imaginary parts of the root vectors corresponding to the non-annihilating roots of $X$. It is known that $\operatorname{dim} O_{X}=\left|\mathcal{N}_{X}\right|$ [16, VI.4]. Indeed, the tangent space at $X$ to $O_{X}$ is spanned by the vectors in $\mathcal{N}_{X}$ and these are linearly independent (see the proof of Prop. 11).

2.3. Type of an Element. The torus of $s u(n)$, the classical Lie algebra of type $A_{n-1}$ (or type $S U(n)$ ) consists of the diagonal matrices in $s u(n)$. After applying a suitable Weyl conjugate, any $X$ in the torus can be identified with the $n$-vector of the real parts of the diagonal elements,

$$
X=(\underbrace{a_{1}, \ldots, a_{1}}_{s_{1}}, \ldots, \underbrace{a_{m}, \ldots, a_{m}}_{s_{m}}),
$$

where the $a_{j} \in \mathbb{R}$ are distinct and $\sum_{j=1}^{m} s_{j} a_{j}=0$. This means that $i a_{j}$ is an eigenvalue of the $n \times n$ matrix $X$ with multiplicity $s_{j}$. The set of annihilating roots 
of $X$ is $\Phi_{X}=\Psi_{1} \cup \cdots \cup \Psi_{m}$ where

$$
\begin{aligned}
& \Psi_{1}=\left\{e_{i}-e_{j}: 1 \leq i \neq j \leq s_{1}\right\} \text { and } \\
& \Psi_{l}=\left\{e_{i}-e_{j}: s_{1}+\cdots+s_{l-1}<i \neq j \leq s_{1}+\cdots+s_{l}\right\} \text { for } l>1 .
\end{aligned}
$$

Following 9, we say that $X$ is type $S U\left(s_{1}\right) \times \cdots \times S U\left(s_{m}\right)$ as this is the Lie type of its set of annihilating roots.

The torus of $s o(2 n+1)$, the classical Lie algebra of type $B_{n}$, consists of block diagonal matrices, with $n 2 \times 2$ blocks of the form $\left[\begin{array}{cc}0 & b_{j} \\ -b_{j} & 0\end{array}\right]$ having $b_{j} \geq 0$, and a 0 in the final diagonal position. We identify $X$ in the torus with the $n$-vector $\left(b_{1}, \ldots, b_{n}\right) \in \mathbb{R}^{+n}$. Up to a Weyl conjugate, $X$ can thus be identified with the $n$-vector

$$
X=(\underbrace{0, \ldots, 0}_{J}, \underbrace{a_{1}, \ldots, a_{1}}_{s_{1}}, \ldots, \underbrace{a_{m}, \ldots, a_{m}}_{s_{m}})
$$

where the $a_{j}>0$ are distinct. One can see that 0 is an eigenvalue of the $(2 n+$ $1) \times(2 n+1)$ matrix $X$ with multiplicity $2 J+1$ and $\pm i a_{j}$ are eigenvalues with multiplicity $s_{j}$.

The set of annihilating roots $\Phi_{X}=\Psi_{0} \cup \Psi_{1} \cdots \cup \Psi_{m}$ where

$$
\begin{aligned}
& \Psi_{0}=\left\{ \pm e_{k}, \pm e_{i} \pm e_{j}: 1 \leq i, j, k \leq J, i \neq j\right\} \text { and } \\
& \Psi_{l}=\left\{e_{i}-e_{j}: J+s_{1}+\cdots+s_{l-1}<i \neq j \leq J+s_{1}+\cdots+s_{l}\right\}
\end{aligned}
$$

for $l=1, \ldots, m$. We will say that $X$ is type

$$
B_{J} \times S U\left(s_{1}\right) \times \cdots \times S U\left(s_{m}\right),
$$

as this is the Lie type of $\Phi_{X}$. Here by $B_{1}$ we mean the root subsystem $\left\{ \pm e_{1}\right\}$, while $S U(1), B_{0}$ and $S U(0)$ are empty (and typically omitted in the description).

Similarly, if $X$ belongs to the torus of the Lie algebra of type $C_{n}$ or $D_{n}$ then, up to a Weyl conjugate, $X$ can be identified with the $n$-vector

$$
X=(\underbrace{0, \ldots, 0}_{J}, \underbrace{a_{1}, \ldots, a_{1}}_{s_{1}}, \ldots, \underbrace{a_{m}, \ldots,( \pm) a_{m}}_{s_{m}})
$$

where the $a_{j}>0$ are distinct. We remark that the minus sign is needed only in type $D_{n}$ and only if $J=0$. (This is because the Weyl group in type $D_{n}$ changes only an even number of signs.) Viewing $X$ as an $2 n \times 2 n$ matrix in $s p(n)$ or $s o(2 n)$, this means that 0 is an eigenvalue of $X$ with multiplicity $2 J$, and $\pm i a_{j}$ are eigenvalues with multiplicity $s_{j}$.

The set of annihilating roots of $X$ can again be written as $\Phi_{X}=\Psi_{0} \cup \Psi_{1} \cdots \cup$ $\Psi_{m}$. In this case

$$
\Psi_{0}=\left\{ \pm 2 e_{k}, \pm e_{i} \pm e_{j}: 1 \leq i, j, k \leq J, i \neq j\right\}
$$

when the Lie algebra is type $C_{n}$ and

$$
\Psi_{0}=\left\{ \pm e_{i} \pm e_{j}: 1 \leq i, j \leq J, i \neq j\right\}
$$

when the Lie algebra is type $D_{n}$. For $l \geq 1$, the $\Psi_{l}$ are as in type $B_{n}$, except when $X=\left(a_{1}, \ldots, a_{1}, \ldots, a_{m}, \ldots,-a_{m}\right)$ in $D_{n}$ when

$$
\Psi_{m}=\left\{ \pm\left(e_{i}-e_{j}\right), \pm\left(e_{i}+e_{n}\right): n-s_{m}<i \neq j \leq n-1\right\} .
$$

We will say $X$ is type

$$
C_{J} \times S U\left(s_{1}\right) \times \cdots \times S U\left(s_{m}\right) \text { or } D_{J} \times S U\left(s_{1}\right) \times \cdots \times S U\left(s_{m}\right)
$$


respectively, as these are the Lie types of $\Phi_{X}$. Here $C_{1}$ is the subsystem $\left\{ \pm 2 e_{1}\right\}$, $C_{2}$ is $\left\{ \pm 2 e_{1}, \pm 2 e_{2}, \pm e_{1} \pm e_{2}\right\}, D_{2}$ is $\left\{ \pm e_{1} \pm e_{2}\right\}$ (or type $A_{1} \times A_{1}$ ), $D_{3}$ is defined in the obvious way, and $D_{1}, D_{0}, C_{0}$ are empty (and often omitted).

Note that there are two distinct subsystems (up to Weyl conjugacy) of annihilating roots of elements of type $S U(n)$ in $D_{n}$.

Definition 1. Suppose $X$ is in the torus of the Lie algebra of type $B_{n}$ and is type $B_{J} \times S U\left(s_{1}\right) \times \cdots \times S U\left(s_{m}\right)$. We will say $X$ is dominant $B$ type if $2 J \geq \max s_{j}$, and is dominant $S U$ type otherwise. We define dominant $C$ and $D$ type similarly for $X$ in $C_{n}$ or $D_{n}$.

It was shown in [9, Thm. 8.2] that for each non-zero $X \in \mathfrak{g}_{n}$, there is an integer $k(X)$ such that for $k \geq k(X), \mu_{X}^{k} \in L^{1} \cap L^{2}\left(\mathfrak{g}_{n}\right)$ (in particular, $\mu_{X}^{k}$ is absolutely continuous with respect to Lebesgue measure) and $\mu_{X}^{k}$ is purely singular if $k<k(X)$. A formula was given for $k(X)$ depending only on the type of $X$ and the type of the Lie algebra. For example, if $X$ is dominant $S U$ type in the Lie algebra of type $B_{n}, C_{n}$ or $D_{n}$, and not of type $S U(n)$ when the Lie algebra is type $D_{n}$, then $k(X)=2$. If $X$ is type $B_{n-1},\left(C_{n-1}, D_{n-1}\right.$ or $\left.S U(n-1)\right)$ in the Lie algebra of type $B_{n}\left(C_{n}, D_{n}\right.$ or $\left.S U(n)\right)$, then $k(X)=n$ and this is the maximal choice required for $k(X)$.

\section{Statement of the Main Result}

3.1. Eligible and Exceptional Tuples. We introduce the following terminology.

Notation 1 . If $X$ is of type $S U\left(s_{1}\right) \times \cdots \times S U\left(s_{m}\right)$ in the Lie algebra of type $A_{n}$, put $S_{X}=\max s_{j}$.

If $X$ is type $B_{J} \times S U\left(s_{1}\right) \times \cdots \times S U\left(s_{m}\right)$ in the Lie algebra of type $B_{n}$, put

$$
S_{X}= \begin{cases}2 J & \text { if } X \text { is dominant } B \text { type } \\ \max s_{j} & \text { else }\end{cases}
$$

Define $S_{X}$ similarly when $X$ belongs to the Lie algebras of type $C_{n}$ or $D_{n}$.

If $X \in s o(2 n+1)$ is dominant $B$ type, then the dimension of the largest eigenspace of the matrix $X$ is $S_{X}+1$, while if $X$ is dominant $S U$ type, then the dimension of the largest eigenspace is $S_{X}$. In all the other Lie algebras, $S_{X}$ is the dimension of the largest eigenspace when $X$ is viewed as a matrix in the appropriate classical matrix algebra.

Definition 2. (i) We will say that the $L$-tuple $\left(X_{1}, X_{2}, \ldots, X_{L}\right)$ of elements in the torus of a Lie algebra of type $S U(n+1)$ is eligible in $\mathfrak{g}_{n}$ if

$$
\sum_{i=1}^{L} S_{X_{i}} \leq(L-1)(n+1) .
$$

(ii) We will say that the $L$-tuple $\left(X_{1}, X_{2}, \ldots, X_{L}\right)$ of elements in the torus of a Lie algebra of type $B_{n}, C_{n}$ or $D_{n}$ is eligible in $\mathfrak{g}_{n}$ if

$$
\sum_{i=1}^{L} S_{X_{i}} \leq(L-1) 2 n \text {. }
$$


Definition 3. We will say that $\left(X_{1}, X_{2}, \ldots, X_{L}\right) \in \mathfrak{t}^{L}$ is an exceptional tuple if it is any one of the following:

- $\mathfrak{g}$ is type $S U(2 n), L=2, n \geq 2$ and $X_{1}$ and $X_{2}$ are both of type $S U(n) \times$ $S U(n)$ (i.e., $X_{i}=(\underbrace{a_{i}, \ldots, a_{i}}_{n}, \underbrace{-a_{i}, \ldots,-a_{i}}_{n})$;

- $\mathfrak{g}$ is type $D_{n}, L=2, X_{1}$ is type $S U(n)$ and $X_{2}$ is either type $S U(n)$ or type $S U(n-1)$ (more precisely, type $S U(n-1) \times D_{1}$ or $S U(n-1) \times S U(1)$ );

- $\mathfrak{g}$ is type $D_{4}, L=2, X_{1}$ is type $S U(4)$ and $X_{2}$ is either type $S U(2) \times S U(2)$ and $\Phi_{X_{2}}$ is Weyl conjugate to a subset of $\Phi_{X_{1}}$, or $X_{2}$ is type $S U(2) \times D_{2}$;

- $\mathfrak{g}$ is type $D_{4}, L=3$ and $X_{1}, X_{2}, X_{3}$ are all of type $S U(4)$ with Weyl conjugate sets of annihilators.

Definition 4 . We will call $\left(X_{1}, X_{2}, \ldots, X_{L}\right)$ an absolutely continuous tuple if $\mu_{X_{1}} * \mu_{X_{2}} * \cdots * \mu_{X_{L}}$ is an absolutely continuous measure.

Our main result is that other than for the exceptional tuples, eligibility characterizes absolute continuity of the convolution product. The proof of this theorem will occupy most of the remainder of the paper. Here is the formal statement of the theorem.

\subsection{Main Result.}

THEOREM 1. Let $\mathfrak{g}_{n}$ be one of the classical, compact, connected Lie algebras of type $A_{n}$ with $n \geq 1, B_{n}$ with $n \geq 2, C_{n}$ with $n \geq 3$, or $D_{n}$ with $n \geq 4$. Assume non-zero $X_{i}, i=1,2, \ldots, L$ for $L \geq 2$, belong to the torus of $\mathfrak{g}_{n}$.

(i) Suppose $\left(X_{1}, X_{2}, \ldots, X_{L}\right)$ is not an exceptional tuple. The measure, $\mu_{X_{1}} *$ $\mu_{X_{2}} * \cdots * \mu_{X_{L}}$, is absolutely continuous with respect to Lebesgue measure on $\mathfrak{g}_{n}$ if and only if $\left(X_{1}, X_{2}, \ldots, X_{L}\right)$ is an eligible tuple.

(ii) If $\left(X_{1}, X_{2}, \ldots, X_{L}\right)$ is an exceptional tuple, other than a pair $\left(X_{1}, X_{2}\right)$ of type $(S U(n), S U(n-1)) 1$ in a Lie algebra of type $D_{n}$ with $n \geq 6$, then the measure $\mu_{X_{1}} * \mu_{X_{2}} * \cdots * \mu_{X_{L}}$ is not absolutely continuous.

REMARK 1. The characterization of absolute continuity in type $A_{n}$ was previously established by Wright $\mathbf{2 3}$. We will include a proof in this paper as our approach is completely different and requires little additional effort.

REMARK 2. (i) We conjecture that a pair of type $(S U(n), S U(n-1))$ in $D_{n}$ with $n \geq 6$ also fails to be absolutely continuous.

(ii) Notice that unlike the case for convolutions of the same orbital measure ([9, Thm. 8.2]), the property of being absolutely continuous does not depend only upon the type of the annihilating root systems of the underlying elements, but also, in some cases, upon their Weyl conjugacy class.

In proving both absolute continuity and its failure we will rely crucially upon the following known geometric properties.

The notation $T_{Z}\left(O_{X}\right)$ will denote the tangent space to $O_{X}$ at $Z \in O_{X}$.

Proposition 1. The measure $\mu_{X_{1}} * \mu_{X_{2}} * \cdots * \mu_{X_{L}}$ on $\mathfrak{g}_{n}$ is absolutely continuous with respect to Lebesgue measure if and only if any of the following hold:

(i) $\sum_{i=1}^{L} O_{X_{i}} \subseteq \mathfrak{g}_{n}$ has non-empty interior;

\footnotetext{
$* *$.

${ }^{1}$ When we say a pair $(X, Y)$ is of type $(*, * *)$ we mean that $X$ is of type $*$ and $Y$ is of type
} 
(ii) $\sum_{i=1}^{L} O_{X_{i}} \subseteq \mathfrak{g}_{n}$ has positive Lebesgue measure;

(iii) There exists $g_{i} \in G_{n}$ with $g_{1}=I d$, such that

$$
\operatorname{sp}\left\{\operatorname{Ad}\left(g_{i}\right)\left(\mathcal{N}_{X_{i}}\right): i=1, \ldots, L\right\}=\mathfrak{g}_{n},
$$

(iv) There exists $g_{i} \in G_{n}$ with $g_{1}=I d$, such that

$$
\sum_{i=1}^{L} T_{A d\left(g_{i}\right)\left(X_{i}\right)}\left(O_{X_{i}}\right)=\mathfrak{g}_{n} .
$$

Furthermore, if the identity holds in (iii) or (iv) for one choice of $\left(g_{2}, \ldots, g_{L}\right) \in$ $G_{n}^{L-1}$, then it holds for all $\left(g_{2}, \ldots, g_{L}\right)$ in an open dense subset of $G_{n}^{L-1}$ of full measure.

REMARK 3. We note that (ii) implies that if $\mu_{X_{1}} * \mu_{X_{2}} * \cdots * \mu_{X_{L}}$ is not absolutely continuous, then $\mu_{X_{1}} * \mu_{X_{2}} * \cdots * \mu_{X_{L}}$ is a purely singular measure.

PROOF. This proposition is a compilation of arguments that can be found in 6, 9 and [8]. We include a sketch here for the convenience of the reader. We will show that (iii) and (iv) are equivalent and then demonstrate the implications (ii) $\Rightarrow$ (iv) $\Rightarrow$ absolute continuity and (iv) $\Rightarrow$ (i). If $\mu_{X_{1}} * \mu_{X_{2}} * \cdots * \mu_{X_{L}}$ is absolutely continuous or (i) holds, then (ii) clearly holds so this completes the equivalence.

(iii) $\Leftrightarrow$ (iv). It is well known (see [6, [16, VI.4]) that

$$
T_{X}\left(O_{X}\right)=\left\{[Y, X]: Y \in \mathfrak{g}_{n}\right\} .
$$

Writing $Y=\sum a_{\alpha} R E_{\alpha}+b_{\alpha} I E_{\alpha}+t$ for some $t \in \mathfrak{t}_{n}$ and $a_{\alpha}, b_{\alpha}$ real, it is easily seen that $T_{X}\left(O_{X}\right)=s p \mathcal{N}_{X}$. Further, $T_{A d(g) X}\left(O_{x}\right)=A d(g)\left(T_{X}\left(O_{X}\right)\right)=\operatorname{sp}\left\{A d(g) \mathcal{N}_{x}\right\}$, proving the equivalence of (iii) and (iv).

The final comment is an analyticity argument. Assume (iii) holds, for example, with $g=\left(I d, g_{2}, \ldots, g_{L}\right)$. For any $h=\left(h_{1}, h_{2}, \ldots, h_{L}\right) \in G_{n}^{L}, h_{1}=I d$, consider the collection $A d\left(h_{j}\right) Y$ for $Y \in \mathcal{N}_{X_{j}}$ and $j=1, \ldots, L$, as vectors in $\mathbb{R}^{\operatorname{dim} \mathfrak{g}_{n}}$, and form the associated matrix $M(h)$. As (iii) holds with $g$, there is a suitable square submatrix of $M(g)$ with non-zero determinant. By analyticity of the determinant map, the determinant of the corresponding square submatrix of $M(h)$ must be nonzero for an open, dense subset of $h \in G_{n}^{L-1}$ of full measure. The same argument applies to (iv).

(ii) $\Rightarrow$ (iv). Consider the addition map $F: O_{X_{1}} \times \cdots \times O_{X_{L}} \rightarrow \mathfrak{g}_{n}$ given by $F\left(Y_{1}, \ldots, Y_{L}\right)=\sum_{j=1}^{L} Y_{j}$. The image of $F$ is $\sum_{j=1}^{L} O_{X_{j}}$. If the rank of $F$ is not full at any point in its domain, then Sard's theorem ([13. p. 286]) implies the measure of the image of $F$ is zero. Thus the differential of $F$ at some point $Y=\left(Y_{1}, \ldots, Y_{L}\right)$, where $Y_{j}=A d\left(g_{j}\right) X_{j}$, has full rank. But the range of the differential of $F$ at $Y$ is $\sum_{j=1}^{L} T_{Y_{j}}\left(O_{X_{j}}\right)$ and hence this sum must be $\mathfrak{g}_{n}$.

(iv) $\Rightarrow$ (i). The hypothesis of (iv) guarantees that the map $F$ defined above has full rank at some point $Y$. By the Implicit function theorem, $F$ is an open map in a neighbourhood of $Y$ and thus Im $F$ has non-empty interior.

(iv) $\Rightarrow$ absolute continuity. This is similar again. To see that the measure $\mu=\mu_{X_{1}} * \mu_{X_{2}} * \cdots * \mu_{X_{L}}$ is absolutely continuous with respect to $m$, we should show that $\mu(E)=0$ whenever $m(E)=0$. Define $f: G_{n}^{L} \rightarrow \mathfrak{g}_{n}$ by

$$
f\left(g_{1}, \ldots, g_{L}\right)=F\left(A d\left(g_{1}\right) X_{1}, \ldots, A d\left(g_{L}\right) X_{L}\right) .
$$

By definition, $\mu(E)=m_{G_{n}^{L}}\left(f^{-1}(E)\right)$. By (iv), the differential of $f$ has full rank at some point. An analyticity argument ensures that this is true on a subset of $g \in G_{n}^{L}$ 
of full measure. An application of the Implicit function theorem shows $f^{-1}(E)$ has $m_{G_{n}^{L}}$ - measure zero. For more details see [18, Thm. 2.2].

An immediate corollary of this proposition and the main theorem is the following.

Corollary 1. Suppose $\left(X_{1}, X_{2}, \ldots, X_{L}\right)$ is eligible and not exceptional. Then $\sum_{i=1}^{L} O_{X_{i}}$ has non-empty interior. If $\left(X_{1}, X_{2}, \ldots, X_{L}\right)$ is either not eligible or is exceptional and not type $(S U(n), S U(n-1))$ in $D_{n}$, then $\sum_{i=1}^{L} O_{X_{i}}$ has measure zero.

There is a sufficient condition for absolute continuity, established by Wright in 23. that we will use in the proof of the main theorem to establish the absolute continuity of certain convolution products of orbital measures in small rank Lie algebras. We state this result below. By the rank of a subsystem we mean the dimension of the vector space it spans.

Theorem 2. [23, Thm. 1.3] Let $X_{1}, \ldots, X_{L}$ belong to the torus of $\mathfrak{g}_{n}$. Assume

$$
(L-1)(|\Phi|-|\Psi|)-1 \geq \sum_{i=1}^{L}\left(\left|\Phi_{X_{i}}\right|-\min _{\sigma \in W}\left|\Phi_{X_{i}} \cap \sigma(\Psi)\right|\right)
$$

for all root subsystems $\Psi \subseteq \Phi$ of rank $n-1$ and having the property that $\operatorname{sp}(\Psi) \cap \Phi=$ $\Psi$. Then $\mu_{X_{1}} * \cdots * \mu_{X_{L}}$ is absolutely continuous.

\section{Tuples That Are Not Absolutely Continuous}

We begin by establishing the necessity of the conditions which give absolute continuity.

\subsection{Eligibility is a requirement for absolute continuity.}

LEMma 1. If $\left(X_{1}, \ldots, X_{L}\right)$ is an absolutely continuous $L$-tuple, then $\left(X_{1}, \ldots, X_{L}\right)$ is eligible.

Proof. Suppose the $L$-tuple, $\left(X_{1}, \ldots, X_{L}\right) \in \mathfrak{g}_{n}^{L}$, is not eligible, that is,

$$
\sum_{i=1}^{L} S_{X_{i}} \geq(L-1) 2 n+1\left(\text { or }(L-1)(n+1)+1 \text { if } \mathfrak{g}_{n} \text { is type } A_{n} .\right)
$$

Let $\alpha_{i}$ be the eigenvalue of $X_{i}$ with greatest multiplicity (where we view each $X_{i}$ as a complex matrix of the appropriate size depending on the Lie type of $\mathfrak{g}_{n}$ ) and let $g_{i}$ belong to the associated Lie group, $G_{n}$. Let $V_{i}$ be the eigenspace of $A d\left(g_{i}\right)\left(X_{i}\right)$ corresponding to the eigenvalue $\alpha_{i}$.

If $\mathfrak{g}_{n}$ is of type $C_{n}$ or $D_{n}$, then $A d\left(g_{i}\right)\left(X_{i}\right)$ are $2 n \times 2 n$ matrices and $\operatorname{dim} V_{i}=$ $S_{X_{i}}$, so

$$
\sum_{i=1}^{L} \operatorname{dim} V_{i}=\sum_{i=1}^{L} S_{X_{i}} \geq(L-1) 2 n+1
$$


We deduce that

$$
\begin{aligned}
& \operatorname{dim} \bigcap_{i=1}^{L} V_{i} \\
= & \sum_{i=1}^{L} \operatorname{dim} V_{i}-\left(\operatorname{dim}\left(V_{1}+V_{2}\right)+\operatorname{dim}\left(\left(V_{1} \cap V_{2}\right)+V_{3}\right)+\cdots+\operatorname{dim}\left(\bigcap_{i=1}^{L-1} V_{i}+V_{L}\right)\right) \\
\geq & (L-1) 2 n+1-2 n(L-1) \geq 1,
\end{aligned}
$$

and hence the matrices, $\operatorname{Ad}\left(g_{i}\right)\left(X_{i}\right)$, have a common eigenvector, $v$. As

$$
\sum_{i=1}^{L} A d\left(g_{i}\right)\left(X_{i}\right)(v)=\sum_{i=1}^{L} \alpha_{i} v,
$$

it follows that $\sum_{i} \alpha_{i}$ is an eigenvalue of $\sum_{i} A d\left(g_{i}\right)\left(X_{i}\right)$. Since $\sum_{i} A d\left(g_{i}\right)\left(X_{i}\right)$ is an arbitrary element of $O_{X_{1}}+\cdots+O_{X_{L}}$, one can see that every element of $\sum_{i} O_{X_{i}}$ has eigenvalue $\sum_{i} \alpha_{i}$. This is impossible if $O_{X_{1}}+\cdots+O_{X_{L}}$ has non-empty interior, thus an application of Prop. 1(i) allows us to conclude that $\mu_{X_{1}} * \cdots * \mu_{X_{L}}$ is not absolutely continuous.

The argument is similar if $\mathfrak{g}_{n}$ is type $A_{n}$, viewing $X_{i}$ as matrices in $s u(n+1)$, acting on $\mathbb{R}^{n+1}$.

In the case when $\mathfrak{g}_{n}$ is type $B_{n}$ we require a slight variation on the argument since every matrix in the Lie algebra $s o(2 n+1)$ (the model for type $B_{n}$ ) has 0 as an eigenvalue. We use the same notation as above and first observe that if all $X_{i}$ are dominant $B$ type, then all $\alpha_{i}=0$ and $\operatorname{dim} V_{i}=S_{X_{i}}+1$. Thus $\sum_{i=1}^{L} \operatorname{dim} V_{i} \geq(L-1) 2 n+L+1$. Since the vector spaces $V_{i}$ are subspaces of $\mathbb{R}^{2 n+1}$, it follows that

$$
\operatorname{dim} \bigcap_{i=1}^{L} V_{i} \geq(L-1) 2 n+L+1-(2 n+1)(L-1) \geq 2 .
$$

Consequently, 0 is an eigenvalue of every element of $O_{X_{1}}+\cdots+O_{X_{L}}$ of multiplicity at least two. Again, we can conclude that $O_{X_{1}}+\cdots+O_{X_{L}}$ has empty interior and therefore $\left(X_{1}, \ldots, X_{L}\right)$ is not an absolutely continuous tuple.

If, instead, precisely one $X_{i}$ is dominant $S U$ type, with eigenvalue $\alpha \neq 0$ of maximum multiplicity, then $\sum \operatorname{dim} V_{i} \geq(L-1) 2 n+L$. This shows that $\operatorname{dim} \bigcap_{i=1}^{L} V_{i}$ has dimension at least one and hence every element of $O_{X_{1}}+\cdots+O_{X_{L}}$ has $\alpha$ as an eigenvalue, again a contradiction if $\left(X_{1}, \ldots, X_{L}\right)$ is an absolutely continuous tuple.

If two or more $X_{i}$ are dominant $S U$ type, then $\left(X_{1}, \ldots, X_{L}\right)$ is automatically eligible.

\subsection{Exceptional tuples that are not absolutely continuous.}

Lemma 2. Suppose $\left(X_{1}, \ldots, X_{L}\right)$ is an exceptional tuple and is not a pair $\left(X_{1}, X_{2}\right)$ of type $(S U(n), S U(n-1))$ in $D_{n}$ where $n \geq 6$. Then $\left(X_{1}, \ldots, X_{L}\right)$ is not an absolutely continuous tuple.

Proof. We will need separate arguments for the various exceptional tuples.

(i) Suppose $X_{1}$ and $X_{2}$ are both type $S U(n)$ in the Lie algebra $D_{n}$. Observe that

$$
\operatorname{dim}\left(\operatorname{sp}\left\{\operatorname{Ad}\left(g_{i}\right)\left(\mathcal{N}_{X_{i}}\right): i=1,2\right\}\right) \leq\left|\mathcal{N}_{X_{1}}\right|+\left|\mathcal{N}_{X_{2}}\right| .
$$


In this case, $\left|\mathcal{N}_{X_{i}}\right|=\left|\Phi_{n}\right| / 2$. As the dimension of the Lie algebra is $\left|\Phi_{n}\right|+n$ it is clearly impossible for $\operatorname{sp}\left\{A d\left(g_{i}\right)\left(\mathcal{N}_{X_{i}}\right): i=1,2\right\}$ to be the full Lie algebra. Thus Prop. 1(iii) proves that this pair is not absolutely continuous.

(ii) Suppose $X_{1}$ and $X_{2}$ are of types $S U(n)$ and $S U(n-1)$, respectively, in $D_{n}$ with $n=4$ or 5 . For this problem, we will use the fact that a root system of type $S U(4)$ is isomorphic to one of type $D_{3}$. We will explain the argument for $n=4$ and leave $n=5$ as an exercise.

Let $\pi$ be an automorphism of the root system of type $D_{4}$ (an isomorphism that preserves the Cartan matrix) that maps the annihilating roots of $X_{1}$ (those of type $S U(4)$ ) onto a root subsystem of type $D_{3}$. This automorphism extends to an automorphism on the torus of $D_{4}$ which maps $X_{1}$ to the element $\pi\left(X_{1}\right)$ whose set of annihilating roots is the $D_{3}$ root subsystem, and it maps $X_{2}$ to the element $\pi\left(X_{2}\right)$ whose set of annihilating roots is isomorphic to those of $X_{2}$ and hence is type $S U(3)$ (as this is unique up to Lie isomorphism). It induces a Lie algebra isomorphism that we also call $\pi$. We have $\pi\left(O_{X_{j}}\right)=O_{\pi\left(X_{j}\right)}$ and

$$
\pi\left(T_{A d\left(g_{j}\right)\left(X_{j}\right)}\left(O_{X_{j}}\right)\right)=T_{A d\left(\pi\left(g_{j}\right)\right)\left(\pi\left(X_{j}\right)\right)}\left(O_{\pi\left(X_{j}\right)}\right)
$$

where if $g_{j}=\exp H_{j}$, then $\pi\left(g_{j}\right)=\exp \pi\left(H_{j}\right)$.

The pair $\left(\pi\left(X_{1}\right), \pi\left(X_{2}\right)\right)$ is not eligible in $D_{4}$ as $S_{\pi\left(X_{1}\right)}=6$ and $S_{\pi\left(X_{2}\right)}=3$, so by our previous lemma it is not an absolutely continuous pair. Consequently, Prop. 1(iv) implies that

$$
\operatorname{dim}\left(\sum_{i=1}^{2} T_{A d\left(\pi\left(g_{i}\right)\right) \pi\left(X_{i}\right)}\left(O_{\pi\left(X_{i}\right)}\right)\right)<\operatorname{dim} D_{n}
$$

for any choices of $g_{1}, g_{2}$. But then a similar statement holds for $\sum_{i=1}^{2} T_{A d\left(g_{j}\right) X_{j}}\left(O_{X_{j}}\right)$ and thus $\left(X_{1}, X_{2}\right)$ is not an absolutely continuous pair.

(iii) When $\left(X_{1}, X_{2}\right)$ is a pair of type $\left(S U(4), S U(2) \times D_{2}\right)$ in $D_{4}$ the arguments are similar. The Lie isomorphism, $\pi$, that maps the subsystem of type $S U$ (4) onto one of type $D_{3}$ must preserve the type of the root subsystem of type $S U(2) \times D_{2}$. But the pair $\left(\pi\left(X_{1}\right), \pi\left(X_{2}\right)\right)$ is not eligible and hence neither it, nor the original pair, can be absolutely continuous.

Next, suppose $X_{1}$ is type $S U(4)$ and $X_{2}$ is type $S U(2) \times S U(2)$ in $D_{4}$ with the subsystem, $\Phi_{X_{2}}$, Weyl conjugate to a subset of the subsystem $\Phi_{X_{1}}$. Since any Weyl conjugate of $X_{2}$ generates the same orbit as $X_{2}$ there is no loss of generality in assuming $\Phi_{X_{2}} \subseteq \Phi_{X_{1}}$. Consider the same Lie isomorphism $\pi$ again. Then $\pi\left(\Phi_{X_{2}}\right) \subseteq \pi\left(\Phi_{X_{1}}\right)$ has the same Lie type as $\Phi_{X_{2}}$. But the only subsystems of type $D_{3}$ that are isomorphic to type $S U(2) \times S U(2)$ are of the form $\left\{ \pm e_{i} \pm e_{j}\right\}$ for some $i \neq j$, and hence are type $D_{2}$. Being of type $\left(D_{3}, D_{2}\right)$, the pair $\left(\pi\left(X_{1}\right), \pi\left(X_{2}\right)\right)$ is not eligible and therefore $\left(X_{1}, X_{2}\right)$ is not absolutely continuous.

(iv) Assume $X_{1}, X_{2}, X_{3}$ are each of type $S U(4)$ in $D_{4}$, with Weyl conjugate sets of annihilators. As the annihilators are Weyl conjugate, for each $i=1,2,3$ there exist $h_{i}$ in the Lie group of type $D_{4}$ such that $\operatorname{Ad}\left(h_{i}\right)\left(\mathcal{N}_{X_{1}}\right)=\mathcal{N}_{X_{i}}$. Therefore there exist $g_{i}$ in the group such that

$$
\operatorname{sp}\left\{\operatorname{Ad}\left(g_{i}\right)\left(\mathcal{N}_{X_{i}}\right): i=1,2,3\right\}=\mathfrak{g}
$$

if and only if

$$
\operatorname{sp}\left\{A d\left(g_{i} h_{i}\right)\left(\mathcal{N}_{X_{1}}\right): i=1,2,3\right\}=\mathfrak{g} .
$$

But the latter was shown to be impossible in the proof of [9. Thm. 8.2]. 
(v) The argument is similar if $X_{1}$ and $X_{2}$ are both type $S U(n) \times S U(n)$ in the Lie algebra of type $S U(2 n)$. In this case, $\mathcal{N}_{X_{1}}$ and $\mathcal{N}_{X_{2}}$ are Weyl conjugate and it was shown in [9. Prop. 5.1] that there is no $g \in S U(2 n)$ such that $s p\left\{A d(g) \mathcal{N}_{X_{1}}, \mathcal{N}_{X_{1}}\right\}=$ $s u(2 n)$.

\section{Proving Absolute Continuity - Main Ideas}

5.1. General Strategy. Our proof that the eligible, non-exceptional tuples are absolutely continuous will proceed by induction on the rank of the Lie algebra. The reduction is based upon the following idea.

Notation 2. Suppose $X$ in the torus of the Lie algebra of type $S U(n), B_{n}$, $C_{n}$ or $D_{n}$ is identified (after a suitable Weyl conjugate) with the $n$-vector

$$
(\underbrace{0, \ldots, 0}_{J}, \underbrace{a_{1}, \ldots, a_{1}}_{s_{1}}, \ldots, \underbrace{a_{m}, \ldots,( \pm) a_{m}}_{s_{m}})
$$

where $s_{1}=\max s_{j}$ and $J=0$ in the case of type $S U(n)$. Define the element $X^{\prime} \in \mathfrak{t}_{n-1}$ by

$$
X^{\prime}= \begin{cases}(\underbrace{0, \ldots, 0}_{J-1}, \underbrace{a_{1}, \ldots, a_{1}}_{s_{1}}, \ldots, \underbrace{a_{m}, \ldots,( \pm) a_{m}}_{s_{m}}) & \text { if } 2 J \geq s_{1} \\ (\underbrace{0, \ldots, 0}_{J}, \underbrace{a_{1}, \ldots, a_{1}}_{s_{1}-1}, \ldots, \underbrace{a_{m}, \ldots,( \pm) a_{m}}_{s_{m}}) & \text { if } 2 J<s_{1}\end{cases}
$$

This means, for example, that if $X$ has type $B_{J} \times S U\left(s_{1}\right) \times \cdots \times S U\left(s_{m}\right)$ where $s_{1}=\max s_{j}$, then $X^{\prime}$ has type $B_{J-1} \times S U\left(s_{1}\right) \times \cdots \times S U\left(s_{m}\right)$ if $X$ is dominant $B$ type and $X^{\prime}$ has type $B_{J} \times S U\left(s_{1}-1\right) \times \cdots \times S U\left(s_{m}\right)$ if $X$ is dominant $S U$ type. If $X$ in $S U(n)$ has type $S U\left(s_{1}\right) \times \cdots \times S U\left(s_{m}\right)$, then $S_{X^{\prime}}=S_{X}-1$ if $s_{1}>\max _{j \geq 2} s_{j}$, and $S_{X^{\prime}}=S_{X}$ otherwise. In the latter case $S_{X} \leq n / 2$.

We can embed $\mathfrak{t}_{n-1}$ into $\mathfrak{t}_{n}$ by taking the standard basis vectors $e_{1}, \ldots, e_{n}$ in $\mathbb{R}^{n}$ (or $e_{1}-e_{n+1}, \ldots, e_{n}-e_{n+1}$ in $\mathbb{R}^{n+1}$ in the case of type $S U(n+1)$ ) as the basis for $\mathfrak{t}_{n}$ and taking the vectors $e_{2}, \ldots, e_{n}$ (resp., $\left.e_{2}-e_{n+1}, \ldots, e_{n}-e_{n+1}\right)$ as the basis for $\mathfrak{t}_{n-1}$. This also gives a natural embedding of $\Phi_{n-1}$ into $\Phi_{n}$ and together these give an embedding of $\mathfrak{g}_{n-1}$ into $\mathfrak{g}_{n}$, an embedding of $\mathcal{V}_{n-1}$ into $\mathcal{V}_{n}$ and an embedding of $G_{n-1}$ into $G_{n}$. We will also view $X^{\prime}$ as an element of $\mathfrak{t}_{n}$ in the natural way.

An induction argument will be applicable because of the following lemma.

Lemma 3. If $(X, Y)$ is an eligible pair in $\mathfrak{g}_{n}$ and $X, Y$ are not both of type $S U(m) \times S U(m)$ in the Lie algebra of type $S U(2 m)$, then the reduced pair, $\left(X^{\prime}, Y^{\prime}\right)$, is eligible in $\mathfrak{g}_{n-1}$.

Proof. Case 1: $\mathfrak{g}_{n}$ is type $B_{n}, C_{n}$ or $D_{n}$.

Observe that always $S_{X^{\prime}} \leq S_{X}$ since the dimensions of the eigenspaces of $X^{\prime}$ can only be at most the dimensions of those of $X$.

If both $X$ and $X^{\prime}$ are dominant $B, C$ or $D$ type, then $S_{X^{\prime}}=S_{X}-2$. If $X^{\prime}$ is dominant $S U$ type, then $S_{X^{\prime}} \leq n-1$, regardless of the type of $X$. Finally, if $X$ is dominant $S U$ type while $X^{\prime}$ is dominant $B, C$ or $D$ type, then $S_{X}=s_{1}>$ $2 J=S_{X^{\prime}} \geq s_{1}-1$. Since it is always true that $J+s_{1} \leq n$, one can check that $s_{1} \leq(2 n+1) / 3$ and hence $S_{X^{\prime}} \leq n-1$.

Thus if either $X$ and $X^{\prime}$ or $Y$ and $Y^{\prime}$ are both dominant $B, C$ or $D$ type, then

$$
S_{X^{\prime}}+S_{Y^{\prime}} \leq S_{X}+S_{Y}-2 \leq 2(n-1) .
$$


Otherwise, both $S_{X^{\prime}}$ and $S_{Y^{\prime}} \leq n-1$ and again we conclude that $S_{X^{\prime}}+S_{Y^{\prime}} \leq$ $2(n-1)$.

Case 2: $\mathfrak{g}_{n}$ is type $S U(n+1)$.

If either $S_{X^{\prime}}<S_{X}$ or $S_{Y^{\prime}}<S_{Y}$, then $S_{X^{\prime}}+S_{Y^{\prime}} \leq S_{X}+S_{Y}-1$ and thus $\left(X^{\prime}, Y^{\prime}\right)$ is eligible. Otherwise, $S_{X^{\prime}}=S_{X}$ and $S_{Y^{\prime}}=S_{Y}$ and in that case $S_{X^{\prime}}, S_{Y^{\prime}} \leq$ $(n+1) / 2$. If $n$ is even, then we must have $S_{X^{\prime}}, S_{Y^{\prime}} \leq n / 2$ giving $S_{X^{\prime}}+S_{Y^{\prime}} \leq n$. If $n$ is odd, it is still true that $S_{X^{\prime}}+S_{Y^{\prime}} \leq n$ unless $S_{X^{\prime}}=S_{Y^{\prime}}=(n+1) / 2$. But that happens only when $X$ and $Y$ are both type $S U((n+1) / 2) \times S U((n+1) / 2)$, which is not permitted.

REMARK 4. It is easy to see that if $X$ and $X^{\prime}$ are of opposite dominant types, then $X$ is type $B_{J},\left(C_{J}\right.$ or $\left.D_{J}\right) \times S U\left(s_{1}\right) \times \cdots \times S U\left(s_{m}\right)$ where $1 \leq J<\sum s_{i}$. It follows from [9, Thm. 8.2] that $\mu_{X}^{2} \in L^{2}$.

We record here a well known fact from elementary linear algebra that is a consequence of the continuity of the determinant function and will be quite useful for us.

LEMMA 4. If $\left\{v_{1}, \ldots, v_{n}\right\}$ is a set of linearly independent vectors in vector space $V$ and $w_{1}, \ldots, w_{n} \in V$, then for sufficiently small $\varepsilon>0$, the collection $\left\{v_{1}+\right.$ $\left.\varepsilon w_{1}, \ldots, v_{n}+\varepsilon w_{n}\right\}$ is also linearly independent.

Notation 3. Given $X^{\prime}$ as defined above, let $\mathcal{N}_{X^{\prime}}=\left\{R E_{\alpha}, I E_{\alpha}: \alpha \notin \Phi_{X^{\prime}}\right\}$, (as in 2.5), but viewed as embedded into $\mathcal{V}_{n}$. Let

$$
\Omega_{X}=\mathcal{N}_{X} \backslash \mathcal{N}_{X^{\prime}} .
$$

We will refer to the next result as our general strategy. It will enable us to establish Prop. 1(iii) holds for a given tuple.

Proposition 2. (General Strategy) Let $X_{i} \in \mathfrak{t}_{n}, i=1, \ldots, L$ for $L \geq 2$, and assume $\left(X_{1}^{\prime}, \ldots, X_{L}^{\prime}\right)$ is an absolutely continuous tuple in $\mathfrak{g}_{n-1}$. Suppose $\Omega$ is a subset of $\mathcal{V}_{n} \backslash \mathcal{V}_{n-1}$ that contains all $\Omega_{X_{i}}$ and has the property that ad $(H)(\Omega) \subseteq s p \Omega$ whenever $H \in \mathfrak{g}_{n-1}$. Fix $\Omega_{0} \subseteq \Omega_{X_{L}}$.

Assume there exists $g_{1}, \ldots g_{L-1} \in G_{n-1}$ and $M \in \mathfrak{g}_{n}$ such that

(i) $\operatorname{sp}\left\{\operatorname{Ad}\left(g_{i}\right)\left(\Omega_{X_{i}}\right), \Omega_{X_{L}} \backslash \Omega_{0}: i=1, \ldots, L-1\right\}=\operatorname{sp} \Omega$;

(ii) $a d^{k}(M): \mathcal{N}_{X_{L}} \backslash \Omega_{0} \rightarrow \operatorname{sp}\left\{\Omega, \mathfrak{g}_{n-1}\right\}$ for all positive integers $k$; and

(iii) The span of the projection of $A d(\exp s M)\left(\Omega_{0}\right)$ onto the orthogonal complement of $\operatorname{sp}\left\{\mathfrak{g}_{n-1}, \Omega\right\}$ in $\mathfrak{g}_{n}$ is a surjection for all small $s>0$.

Then $\left(X_{1}, \ldots, X_{L}\right)$ is an absolute continuous tuple.

Proof. As $\left(X_{1}^{\prime}, \ldots, X_{L}^{\prime}\right)$ is an absolutely continuous tuple, Prop. 1(iii) tells us that

$$
\operatorname{sp}\left\{\operatorname{Ad}\left(h_{i}\right)\left(\mathcal{N}_{X_{i}^{\prime}}\right), \mathcal{N}_{X_{L}^{\prime}}: i=1, \ldots, L-1\right\}=\mathfrak{g}_{n-1}
$$

for a dense set of $\left(h_{1}, \ldots, h_{L-1}\right) \in G_{n-1}^{L-1}$. Given $\varepsilon>0$, choose such $h_{i}=h_{i}(\varepsilon) \in$ $G_{n-1}$ with $\left\|A d\left(h_{i}\right)-A d\left(g_{i}\right)\right\|<\varepsilon$, where the elements $g_{i} \in G_{n-1}$ are the ones given in the hypothesis of the proposition. (The norm can be taken to be the operator norm.)

Lemma 4 together with assumption (i), shows that for sufficiently small $\varepsilon>0$,

$$
\begin{aligned}
\operatorname{dim}(\operatorname{sp} \Omega) & =\operatorname{dim}\left(\operatorname{sp}\left\{\operatorname{Ad}\left(g_{i}\right)\left(\Omega_{X_{i}}\right), \Omega_{X_{L}} \backslash \Omega_{0}: i=1, \ldots, L-1\right\}\right) \\
& =\operatorname{dim}\left(\operatorname{sp}\left\{\operatorname{Ad}\left(h_{i}\right)\left(\Omega_{X_{i}}\right), \Omega_{X_{L}} \backslash \Omega_{0}: i=1, \ldots, L-1\right\}\right) .
\end{aligned}
$$


Since $\operatorname{ad}(H)(\Omega) \subseteq \operatorname{sp}(\Omega)$ for all $H \in \mathfrak{g}_{n-1}$ and $h_{i}=\exp H_{i}$ for some $H_{i} \in \mathfrak{g}_{n-1}$ we have

$$
\begin{aligned}
A d\left(h_{i}\right)(\Omega) & =A d\left(\exp H_{i}\right)(\Omega) \\
& =\exp \left(\operatorname{ad}\left(H_{i}\right)(\Omega) \subseteq s p \Omega\right.
\end{aligned}
$$

for all $h_{i} \in G_{n-1}$. Thus for sufficiently small $\varepsilon>0$,

$$
\operatorname{sp}\left\{\operatorname{Ad}\left(h_{i}\right)\left(\Omega_{X_{i}}\right), \Omega_{X_{L}} \backslash \Omega_{0}: L=1, \ldots, L-1\right\}=s p \Omega
$$

For such a choice of $\varepsilon$ (hereafter fixed) we have

$$
\begin{gathered}
\operatorname{sp}\left\{\operatorname{Ad}\left(h_{i}\right)\left(\mathcal{N}_{X_{i}}\right), \mathcal{N}_{X_{L}} \backslash \Omega_{0}: i=1, \ldots, L-1\right\} \\
=\operatorname{sp}\left\{\operatorname{Ad}\left(h_{i}\right)\left(\mathcal{N}_{X_{i}^{\prime}}\right), \operatorname{Ad}\left(h_{i}\right) \Omega_{X_{i}}, \mathcal{N}_{X_{L}^{\prime}}, \Omega_{X_{L}} \backslash \Omega_{0}\right\}=\operatorname{sp}\left\{\Omega, \mathfrak{g}_{n-1}\right\} .
\end{gathered}
$$

Assumption (ii), and the fact that $\mathcal{N}_{X_{L}} \backslash \Omega_{0} \subseteq \operatorname{sp}\left\{\Omega, \mathfrak{g}_{n-1}\right\}$, implies that for any real number $s, \exp (s \cdot a d M)=A d(\exp s M) \operatorname{maps} \mathcal{N}_{X_{L}} \backslash \Omega_{0}$ to $\operatorname{sp}\left\{\Omega, \mathfrak{g}_{n-1}\right\}$. Moreover, $\|I d-A d(\exp s M)\| \rightarrow 0$ as $s \rightarrow 0$, thus similar reasoning to that above shows that for all small enough $s>0$,

$$
\begin{aligned}
\operatorname{sp}\left\{\Omega, \mathfrak{g}_{n-1}\right\} & =\operatorname{sp}\left\{\operatorname{Ad}\left(h_{i}\right)\left(\mathcal{N}_{X_{i}}\right), \mathcal{N}_{X_{L}} \backslash \Omega_{0}: i=1, \ldots, L\right\} \\
& =\operatorname{sp}\left\{\operatorname{Ad}\left(h_{i}\right)\left(\mathcal{N}_{X_{i}}\right),(\operatorname{Ad}(\exp s M))\left(\mathcal{N}_{X_{L}} \backslash \Omega_{0}\right): i=1, \ldots, L-1\right\} .
\end{aligned}
$$

Combined with assumption (iii), this proves that for sufficiently small $s>0$,

$$
s p\left\{\operatorname{Ad}\left(h_{i}\right)\left(\mathcal{N}_{X_{i}}\right), \operatorname{Ad}(\exp s M)\left(\mathcal{N}_{X_{L}}\right): i=1, \ldots, L-1\right\}=\mathfrak{g}_{n} .
$$

Another application of Prop. 1(iii) shows that $\mu_{X_{1}} * \cdots * \mu_{X_{L}}$ is absolutely continuous.

We will occasionally make use of the following specific application of the elementary linear algebra property in order to verify the hypothesis of the general strategy.

Lemma 5. Suppose $\Omega$ is a subset of $\mathcal{V}_{n} \backslash \mathcal{V}_{n-1}$ that contains both $\Omega_{X}$ and $\Omega_{Y}$, and has the property that ad $(H)(\Omega) \subseteq \operatorname{sp} \Omega$ whenever $H \in \mathfrak{g}_{n-1}$. Fix $\Omega_{0} \subseteq \Omega_{X}$. Assume $\Omega_{1} \subseteq\left(\Omega_{Y} \cap \Omega_{X}\right) \backslash \Omega_{0}$ and the vectors in $\left\{\right.$ adH $\left.\left(\Omega_{1}\right), \Omega_{Y} \backslash \Omega_{1}, \Omega_{X} \backslash \Omega_{0}\right\}$ span $\Omega$ for some $H \in \mathfrak{g}_{n-1}$. Then for sufficiently small $t>0$,

$$
s p\left\{A d(\exp t H)\left(\Omega_{Y}\right), \Omega_{X} \backslash \Omega_{0}\right\}=s p \Omega .
$$

Proof. The arguments are similar to that of the general strategy. Since $\left\|a d(H)-\frac{1}{t}(A d(\exp t H)-I d)\right\|$ and $\|I d-A d(\exp t H)\|$ both tend to 0 as $t \rightarrow 0$, and $a d^{k}(H)(\Omega) \subseteq s p \Omega$ for all $k$, the same argument as used above shows that

$$
s p\left\{(A d(\exp t H)-I d)\left(\Omega_{1}\right), A d(\exp t H)\left(\Omega_{Y} \backslash \Omega_{1}\right), \Omega_{X} \backslash \Omega_{0}\right\}=s p \Omega .
$$

But since $\Omega_{1} \subseteq \Omega_{X} \backslash \Omega_{0}$, we can replace $(A d(\exp t H)-I d)\left(\Omega_{1}\right)$ in the span on the left hand side by $A d(\exp t H)\left(\Omega_{1}\right)$. Hence

$$
s p\left\{A d(\exp t H)\left(\Omega_{Y}\right), \Omega_{X} \backslash \Omega_{0}\right\}=s p \Omega .
$$


5.2. Applying the General strategy with $L=2$. The following proposition, the 'induction step', is the most important ingredient in the proof of the main theorem.

We continue to use the notation $\Omega_{X}=\mathcal{N}_{X} \backslash \mathcal{N}_{X^{\prime}}$, where $X^{\prime}$ was defined in (5.1).

Proposition 3. Suppose $(X, Y)$ is an eligible pair in $\mathfrak{g}_{n}$ other than $X, Y$ both of type $S U(n)$ in $D_{n}$ or type $S U(n / 2) \times S U(n / 2)$ in $S U(n)$. Assume also that the reduced pair, $\left(X^{\prime}, Y^{\prime}\right)$, is an absolutely continuous pair in $\mathfrak{g}_{n-1}$. Then $(X, Y)$ is an absolutely continuous pair in $\mathfrak{g}_{n}$.

Proof. The main task of the proof is to show that any eligible pair, other than one of the two exceptional pairs mentioned, satisfy properties (i) - (iii) of the general strategy, Prop. 2.

Part I: $g_{n}$ is type $B_{n}, C_{n}$ or $D_{n}$.

The proof is divided into three cases depending on the dominant types of $X$ and $Y$.

Case 1: Neither $X$ nor $Y$ are of dominant $S U$ type.

With the notation as before, we have $S_{X}=2 J$ and $S_{Y}=2 K$ (meaning $X$ is dominant $B_{J}\left(C_{J}\right.$ or $\left.D_{J}\right)$ type and $Y$ is dominant $B_{K}\left(C_{K}\right.$ or $\left.D_{K}\right)$ type). Applying a Weyl conjugate, if necessary, we can assume without loss of generality that

$$
\Omega_{X}=\left\{F E e_{1} \pm e_{j}: J<j \leq n, F=R, I\right\}
$$

and similarly

$$
\Omega_{Y}=\left\{F E e_{1} \pm e_{j}: K<j \leq n, F=R, I\right\} .
$$

Case 1(a): $g_{n}$ is type $D_{n}$.

Recall that $\mathcal{V}_{n}$ is set of all real and imaginary parts of the chosen Weyl basis of root vectors of $\mathfrak{g}_{n}$. Put

$$
\Omega=\mathcal{V}_{n} \backslash \mathcal{V}_{n-1}=\left\{F E e_{1} \pm e_{j}: j=2, \ldots, n, F=R, I\right\}
$$

and

$$
\Omega_{0}=\left\{R E e_{1}+e_{n}, I E e_{1}+e_{n}\right\} .
$$

If $H \in \mathfrak{g}_{n-1}$, then $H$ is a linear combination of a torus element of $\mathfrak{g}_{n-1}$ and the vectors $R E e_{i} \pm e_{j}, I E e_{i} \pm e_{j}$ with $2 \leq i<j \leq n$. It follows easily from (2.4) that $\operatorname{ad}(H)(\Omega) \subseteq \operatorname{sp} \Omega$.

Take $g \in G_{n-1}$ to be the Weyl conjugate that permutes the letters $1+j$ and $K+j$ for $j=1, \ldots, J-1$. This is well defined and leaves the letter $n$ unchanged as the eligibility condition ensures $J+K-1 \leq n-1$. Consequently, $A d(g)\left(F E e_{1} \pm\right.$ $\left.e_{K+j}\right)=F E e_{1} \pm e_{1+j}$ for $j=1, \ldots, J-1$, and all other vectors in $\Omega$ are fixed, including $F E e_{1} \pm e_{n}$. Thus

$$
\left\{A d(g)\left(\Omega_{Y}\right), \Omega_{X} \backslash \Omega_{0}\right\}=\left\{F E e_{1} \pm e_{k}: k=2, \ldots, n\right\}
$$

proving that (i) of the general strategy, Prop 2 (with $L=2$ ) is satisfied.

Let $M=R E e_{1}+e_{n} \in \mathfrak{g}_{n}$. Applying (2.4) again, we see that if $H=F E e_{1} \pm e_{j}$ for some $j<n$, then $\operatorname{ad}(M)(H)=c F E e_{j} \mp e_{n} \in \mathfrak{g}_{n-1}$ for a non-zero constant $c$ depending on $j, n$ and $F$. If $H=F E e_{i} \pm e_{n}$, then $\operatorname{ad}(M)(H)=c F E e_{1} \mp e_{i} \in$ $s p\left(\Omega \backslash \Omega_{0}\right)$. Finally, note that $a d(M)(H)=0$ if $H=F E e_{i} \pm e_{j}$ for $1<i, j<n$ or $H=F E e_{1}-e_{n}$. This proves $a d^{k}(M): \mathcal{N}_{X} \backslash \Omega_{0} \rightarrow s p\left\{\Omega, \mathfrak{g}_{n-1}\right\}$ for all positive integers $k$, so that property (ii) of the general strategy is satisfied. 
As $\operatorname{sp}\left\{\Omega, \mathfrak{g}_{n-1}\right\}$ is of co-dimension one, its orthogonal complement is spanned by the projection onto any element in the complement of $s p\left\{\Omega, \mathfrak{g}_{n-1}\right\}$. The torus element,

$$
a d(M)\left(I E e_{1}+e_{n}\right)=\left[R E e_{1}+e_{n}, I E e_{1}+e_{n}\right]:=t_{1}
$$

is such an element. Since

$$
\operatorname{ad}(M)\left(t_{1}\right)=\left[R E e_{1}+e_{n}, t_{1}\right]=c I E e_{1}+e_{n}
$$

for some $c \neq 0$ (see 2.3), it follows that

$$
A d(\exp s M)\left(I E e_{1}+e_{n}\right)=a(s) I E e_{1}+e_{n}+s b(s) t_{1}
$$

where $a(s), b(s) \rightarrow 1$ as $s \rightarrow 0$. Therefore hypothesis (iii) of Prop. 2 is also fulfilled with any $s>0$. Applying that proposition, we conclude that $\mu_{X} * \mu_{Y}$ is absolutely continuous.

Case 1(b): $g_{n}$ is type $B_{n}$.

Again, we will apply the general strategy, but here with

$$
\Omega=\mathcal{V}_{n} \backslash \mathcal{V}_{n-1}=\left\{F E e_{1} \pm e_{j}, F E e_{1}: j=2, \ldots, n, F=R, I\right\}
$$

and

$$
\Omega_{0}=\left\{R E e_{1}+e_{n}, I E e_{1}+e_{n}\right\} .
$$

The fact that $a d(H)(\Omega) \subseteq \Omega$ whenever $H \in \mathfrak{g}_{n-1}$ follows easily from properties of the roots, as with the case $D_{n}$.

For $t>0$, let $g_{t}=\left(\exp t R E e_{n}\right) g$ where $g \in G_{n-1}$ corresponds to the Weyl conjugate that permutes the letters $1+j$ and $K+j$ for $j=1, \ldots, J-1$ as in the previous case. Since $R E e_{n} \in \mathfrak{g}_{n-1}, g_{t} \in G_{n-1}$. Observe that

$$
\left[R E e_{n}, F E e_{1}\right]=c F E e_{1}+e_{n}+c^{\prime} F E e_{1}-e_{n}
$$

and

$$
\left[R E e_{n}, F E e_{1} \pm e_{j}\right]= \begin{cases}c^{( \pm)} F E e_{1} & \text { if } j=n \\ 0 & \text { else }\end{cases}
$$

with $c, c^{\prime}$ and $c^{( \pm)}$non-zero constants. In particular, this implies

$$
A d\left(\exp t R E e_{n}\right)\left(F E e_{1} \pm e_{j}\right)=F E e_{1} \pm e_{j} \text { for } j \neq n .
$$

Since $A d(g)\left(F E e_{1} \pm e_{K+j}\right)=F E e_{1} \pm e_{1+j}$ for $j=1, \ldots, J-1$ and the eligibility condition ensures $A d(g)$ fixes $F E e_{1} \pm e_{n}$, it follows that for $j=1, \ldots, J-1$ we have

$$
\begin{aligned}
A d\left(g_{t}\right)\left(F E e_{1} \pm e_{K+j}\right) & =A d\left(\exp t R E e_{n}\right)\left(F E e_{1} \pm e_{1+j}\right) \\
& =F E e_{1} \pm e_{1+j}
\end{aligned}
$$

and

$$
\begin{aligned}
A d\left(g_{t}\right)\left(F E e_{1} \pm e_{n}\right) & =A d\left(\exp t R E e_{n}\right)\left(F E e_{1} \pm e_{n}\right) \\
& =a(t) F E e_{1} \pm e_{n}+t b(t) F E e_{1}+t^{2} c(t) F E e_{1} \mp e_{n}
\end{aligned}
$$

where $a(t) \rightarrow 1$ as $t \rightarrow 0$, and $b(t)$ and $c(t)$ converge to non-zero scalars. All other choices of $F E e_{1} \pm e_{j}$ are fixed by $A d\left(g_{t}\right)$. Hence

$$
\begin{aligned}
& s p\left\{F E e_{1}-e_{n}, A d\left(g_{t}\right)\left(F E e_{1} \pm e_{n}\right): F=R, I\right\} \\
= & s p\left\{F E e_{1}-e_{n}, F E e_{1}+e_{n}+t b^{\prime}(t) F E e_{1}, F E e_{1}+t c^{\prime}(t) F E e_{1}+e_{n}: F=R, I\right\}
\end{aligned}
$$

${ }^{2} a(t), b(t), c(t)$ depend on $F$ and the choice of \pm , as well as $t$. From here on we will omit noting this dependence, unless it is important. 
where $b^{\prime}(t)$ and $c^{\prime}(t)$ converge to non-zero limits as $t \rightarrow 0$. Since

$$
\left\{F E e_{1} \pm e_{n}, F E e_{1}: F=R, I\right\}
$$

is a set of six linearly independent vectors, so too is the collection

$$
\left\{F E e_{1}-e_{n}, F E e_{1}+e_{n}+t b^{\prime}(t) F E e_{1}, F E e_{1}+t c^{\prime}(t) F E e_{1}+e_{n}: F=R, I\right\}
$$

for sufficiently small $t$, and therefore they span the same space. Because $\Omega_{X} \backslash \Omega_{0}$ contains $F E e_{1}-e_{n}$, it follows that

$$
\begin{aligned}
& s p\left\{A d\left(g_{t}\right)\left(\Omega_{Y}\right), \Omega_{X} \backslash \Omega_{0}\right\} \\
= & s p\left\{A d\left(g_{t}\right)\left(F E e_{1} \pm e_{k}\right), F E e_{1} \pm e_{j}, F E e_{1}-e_{n}: k>K, J<j<n, F=R, I\right\} \\
= & s p\left\{F E e_{1} \pm e_{j}, F E e_{1} \pm e_{n}, F E e_{1}: j \leq n, F=R, I\right\}=s p \Omega .
\end{aligned}
$$

Again, put $M=R E e_{1}+e_{n} \in \mathfrak{g}_{n}$. As with type $D_{n}, a d^{k}(M)\left(F E e_{1} \pm e_{j}\right) \in$ $s p\left\{\mathfrak{g}_{n-1}, \Omega\right\}$ for all $k$ and $j<n$, and $\operatorname{ad}(M)\left(F E e_{1}-e_{n}\right)=0$. Furthermore, $\operatorname{ad}(M)\left(F E e_{j}\right)=0$ if $j \neq 1, n, \operatorname{ad}(M)\left(F E e_{n}\right)=c F E e_{1}$ and $\operatorname{ad}(M)\left(F E e_{1}\right)=$ $c F E e_{n}$, so property (ii) of the general strategy holds. As in the first case, $s p\left\{\mathfrak{g}_{n-1}, \Omega\right\}$ is of co-dimension one in $\mathfrak{g}_{n}$, and just as in type $D_{n}$ property (iii) holds, so we deduce the absolute continuity of $\mu_{X} * \mu_{Y}$ by appealing to Prop. 2,

Case 1(c): $g_{n}$ is type $C_{n}$.

Here we will use a variant on the general strategy. As with type $D_{n}$ we begin with

$$
\Omega=\left\{F E e_{1} \pm e_{j}: j=2, \ldots, n, F=R, I\right\}
$$

and $g$ the Weyl conjugate permuting the letters $1+j$ and $K+j$ for $j=1, \ldots, J-1$. Take

$$
\Omega_{0}=\left\{F E e_{1} \pm e_{n}: F=R, I\right\} .
$$

The eligibility condition gives that $\operatorname{sp}\left\{\operatorname{Ad}(g)\left(\Omega_{Y}\right), \Omega_{X} \backslash \Omega_{0}\right\}=s p \Omega$.

As with type $D_{n}, \operatorname{ad}\left(F E e_{i} \pm e_{j}\right)(\Omega) \subseteq \operatorname{sp}\left\{\Omega, \mathfrak{g}_{n-1}\right\}$ for all $1<i<j \leq n$ and similarly, $\operatorname{ad}\left(F E\left(2 e_{j}\right)\right)(\Omega) \subseteq \Omega$ for $j>1$, so $\operatorname{ad}(H)(\Omega) \subseteq \operatorname{sp}\left\{\Omega, \mathfrak{g}_{n-1}\right\}$ whenever $H \in \mathfrak{g}_{n-1}$. Thus, as in the proof of the general strategy, upon applying the induction assumption we can deduce there is some $h \in G_{n-1}$ such that

$$
\operatorname{sp}\left\{\operatorname{Ad}(h)\left(\mathcal{N}_{Y}\right), \mathcal{N}_{X} \backslash \Omega_{0}\right\}=\operatorname{sp}\left\{\Omega, \mathfrak{g}_{n-1}\right\} .
$$

Once again, we will put $M=R E e_{1}+e_{n} \in \mathfrak{g}_{n}$. As with the types $B_{n}$ and $D_{n}$, standard facts about roots show that $\operatorname{ad}(M)(H) \in \operatorname{sp}\left\{\Omega, \mathfrak{g}_{n-1}\right\}$ for all $H \in \mathcal{N}_{X} \backslash \Omega_{0}$. In fact, for all $k \geq 1, a d^{k}(M)(H) \in \operatorname{sp}\left\{\Omega, \mathfrak{g}_{n-1}\right\}$ for all $H \in \mathcal{N}_{X} \backslash \Omega_{0}$ except for $H=F E\left(2 e_{n}\right)$ as $a d^{k}(M)\left(F E\left(2 e_{n}\right)\right)$ has a component in $F E\left(2 e_{1}\right)$. (Recall that $F E\left(2 e_{n}\right) \in \mathcal{N}_{X}$ since the only roots $2 e_{j} \in \Phi_{X}$ are those with $j \leq J$.) It is because of this exception that we cannot appeal directly to the general strategy.

Another difference between this set up and the situation for types $B_{n}$ and $D_{n}$ is that here $\operatorname{sp}\left\{\Omega, \mathfrak{g}_{n-1}\right\}$ has co-dimension three, its orthogonal complement being spanned by $R E\left(2 e_{1}\right), I E\left(2 e_{1}\right)$ and the projection onto the torus element $\left[R E e_{1}+e_{n}, I E e_{1}+e_{n}\right]$. That will also complicate matters.

Let $\Lambda$ be the subspace spanned by the torus of $\mathfrak{g}_{n-1}$ and the vectors $R E_{\beta}$ and $I E_{\beta}$ where $\beta$ ranges over all the positive roots except $2 e_{1}, 2 e_{n}$,

$$
\Lambda:=\operatorname{sp}\left\{\Omega, \mathfrak{g}_{n-1}\right\} \ominus \operatorname{sp}\left\{R E\left(2 e_{n}\right), I E\left(2 e_{n}\right)\right\} .
$$


Let $\mathcal{P}$ be the orthogonal projection onto $\Lambda$. Since $\mathcal{N}_{X} \backslash\left\{\Omega_{0}, F E\left(2 e_{n}\right)\right\} \subseteq \Lambda$, Property (5.2) implies that

$$
\operatorname{sp}\left\{\mathcal{P}\left(\operatorname{Ad}(h)\left(\mathcal{N}_{Y}\right)\right), \mathcal{N}_{X} \backslash\left\{\Omega_{0}, R E\left(2 e_{n}\right), I E\left(2 e_{n}\right)\right\}\right\}=\Lambda .
$$

Choose $Y_{\beta}^{F}, Y_{j} \in A d(h) \mathcal{N}_{Y}$ and $X_{\beta}^{F}, X_{j} \in \mathcal{N}_{X} \backslash\left\{\Omega_{0}, R E\left(2 e_{n}\right), I E\left(2 e_{n}\right)\right\}$ such that

(i) $Y_{\beta}^{F}+X_{\beta}^{F}=F E_{\beta}+W_{\beta}^{F}$ where $W_{\beta}^{F} \in \operatorname{sp}\left\{R E\left(2 e_{n}\right), I E\left(2 e_{n}\right)\right\}, F=R, I$ and $\beta$ ranges over all roots except $2 e_{1}, 2 e_{n}$, and

(ii) $Y_{j}+X_{j}=t_{j}+W_{j}$ where $j=2, \ldots, n,\left\{t_{2}, \ldots, t_{n}\right\}$ is a basis for $\mathfrak{t}_{n-1}$ and $W_{j} \in \operatorname{sp}\left\{F E\left(2 e_{n}\right)\right\}$.

Note that if we put $t_{1}=\left[R E e_{1}+e_{n}, I E e_{1}+e_{n}\right]$, then $\left\{t_{1}, \ldots, t_{n}\right\}$ is a basis for $\mathfrak{t}_{n}$.

This collection of vectors $\left\{Y_{\beta}^{F}+X_{\beta}^{F}, Y_{j}+X_{j}\right\}$ is linearly independent and hence for small enough $s>0$, so is also the set

$\left\{Y_{\beta}^{F}+A d(\exp s M)\left(X_{\beta}^{F}\right), Y_{j}+A d(\exp s M)\left(X_{j}\right): \beta \neq 2 e_{1}, 2 e_{n}, j=2, \ldots, n, F=R, I\right\}$.

Observe that

$$
\begin{aligned}
Y_{\beta}^{F}+A d(\exp s M)\left(X_{\beta}^{F}\right) & =Y_{\beta}^{F}+X_{\beta}^{F}+(A d(\exp s M)-I d)\left(X_{\beta}^{F}\right) \\
& =F E_{\beta}+W_{\beta}^{F}+s Q_{\beta}^{F}(s),
\end{aligned}
$$

where the vector $Q_{\beta}^{F}(s)$ depends on $s$, but has bounded norm. The projection of $Q_{\beta}^{F}(s)$ onto $s p\left\{R E 2 e_{1}, I E 2 e_{1}\right\}$ is zero since $A d(\exp s M)$ maps $\mathcal{N}_{X} \backslash\left\{\Omega_{0}, F E\left(2 e_{n}\right)\right.$ : $F=R, I\}$ into $\mathfrak{g}_{n} \ominus \operatorname{sp}\left\{R E 2 e_{1}, I E 2 e_{1}\right\}$. Also, it is clear from the definitions that for $\beta \neq e_{1}-e_{n}$, the projection of $F E_{\beta}+W_{\beta}^{F}$ onto $\operatorname{sp}\left\{R E e_{1}-e_{n}, I E e_{1}-e_{n}\right\}$ is zero. Similar statements can be made for $Y_{j}+A d(\exp s M)\left(X_{j}\right)$.

Claim: The collection of vectors, $Y_{\beta}^{F}+A d(\exp s M)\left(X_{\beta}^{F}\right), Y_{j}+A d(\exp s M)\left(X_{j}\right)$ over all positive roots $\beta \neq 2 e_{1}, 2 e_{n}, F=R, I$, and $j=2, \ldots, n$, together with the four vectors $\operatorname{Ad}(\exp s M)\left(F E\left(2 e_{n}\right)\right), A d(\exp s M)\left(F E e_{1}-e_{n}\right)$ for $F=R, I$, are linearly independent.

To prove this we first observe that

$$
\begin{aligned}
{\left[R E e_{1}+e_{n}, F E\left(2 e_{1}\right)\right] } & =c_{1} F E e_{1}-e_{n} \\
{\left[R E e_{1}+e_{n}, F E\left(2 e_{n}\right)\right] } & =c_{2} F E e_{n}-e_{1}, \\
{\left[R E e_{1}+e_{n}, F E e_{1}-e_{n}\right] } & =c_{3} F E\left(2 e_{1}\right)+c_{4} F E\left(2 e_{n}\right)
\end{aligned}
$$

where $c_{j} \neq 0$. Thus

$$
A d(\exp s M)\left(F E e_{1}-e_{n}\right)=a_{s}^{F} F E e_{1}-e_{n}+s b_{s}^{F} F E\left(2 e_{1}\right)+s c_{s}^{F} F E\left(2 e_{n}\right)
$$

and

$$
A d(\exp s M)\left(F E\left(2 e_{n}\right)\right)=s b_{s}^{\prime F} F E e_{1}-e_{n}+s^{2} c_{s}^{\prime F} F E\left(2 e_{1}\right)+a_{s}^{\prime F} F E\left(2 e_{n}\right)
$$

where the coefficients, $a_{s}^{F}, a_{s}^{\prime F}, b_{s}^{F}, b_{s}^{\prime F}, c_{s}^{F}, c_{s}^{\prime F}$, converge to non-zero constants as $s \rightarrow 0$.

The vectors listed in (5.3) and (5.4), as well as those in $\operatorname{sp}\left\{\mathfrak{g}_{n-1}, \Omega\right\}$, belong to $\mathfrak{g}_{n} \ominus s p\left\{t_{1}\right\}$. We view them as vectors in $\mathbb{R}^{d}$ with $d=\operatorname{dim} \mathfrak{g}_{n}-1$, whose coordinates are given by the basis for $\mathfrak{g}_{n} \ominus s p\left\{t_{1}\right\}$ consisting of the torus elements, $\left\{t_{2}, \ldots, t_{n}\right\}$, together with the real and imaginary parts of the Weyl basis $\left\{E_{\alpha}\right\}$, taking as the final six positions the basis vectors $F E e_{1}-e_{n}, F E\left(2 e_{n}\right)$ and $F E\left(2 e_{1}\right), F=R, I$.

With this understanding, consider the square matrix whose rows are given by the vectors $Y_{j}+A d(\exp s M)\left(X_{j}\right)$ for $j=2, \ldots, n$; followed by the vectors 
$Y_{\beta}^{F}+A d(\exp s M)\left(X_{\beta}^{F}\right), \beta \neq 2 e_{1}, 2 e_{n}$, ordered consistently to above so that the final two come from $\beta=e_{1}-e_{n}$; and then finally the four vectors $A d(\exp s M)\left(F E\left(2 e_{n}\right)\right)$ and $A d(\exp s M)\left(F E e_{1}-e_{n}\right)$ (for a small, but fixed, choice of $s$ ).

The calculations above show that this matrix, denoted $A=\left(A_{i j}\right)$, has the form

$$
A=\left[\begin{array}{cccc}
{\left[I_{d-6}+O(s)\right]_{(d-6) \times(d-6)}} & {[O(s)]_{(d-6) \times 2}} & {[*]_{(d-6) \times 2}} & {[0]_{(d-6) \times 2}} \\
{[O(s)]_{2 \times(d-6)}} & {\left[I_{2}+O(s)\right]_{2 \times 2}} & {\left[\begin{array}{cc}
* & * \\
* & *
\end{array}\right]} & {\left[\begin{array}{ll}
0 & 0 \\
0 & 0
\end{array}\right]} \\
{[0]_{2 \times(d-6)}} & {\left[\begin{array}{cc}
s b_{s}^{\prime R} & 0 \\
0 & s b_{s}^{I I}
\end{array}\right]} & {\left[\begin{array}{cc}
a_{s}^{\prime R} & 0 \\
0 & a_{s}^{\prime I}
\end{array}\right]} & {\left[\begin{array}{cc}
O\left(s^{2}\right) & 0 \\
0 & O\left(s^{2}\right)
\end{array}\right]} \\
{[0]_{2 \times(d-6)}} & {\left[\begin{array}{cc}
a_{s}^{R} & 0 \\
0 & a_{s}^{I}
\end{array}\right]} & {\left[\begin{array}{cc}
s c_{s}^{R} & 0 \\
0 & s c_{s}^{I}
\end{array}\right]} & {\left[\begin{array}{cc}
s b_{s}^{R} & 0 \\
0 & s b_{s}^{I}
\end{array}\right]}
\end{array}\right]
$$

where $I_{m}$ denotes the $m \times m$ identity matrix, $O\left(s^{k}\right)$ means terms dominated by $C s^{k}$ for some constant $C$ independent of $s$ and $*$ denotes terms that may depend on $s$, but are bounded independently of $s$.

We estimate the determinant of this matrix using the Leibniz formula: Since $\left|A_{11} A_{22} \cdots A_{d d}\right| \geq C_{0} s^{2}$ for some $C_{0}>0$ and all the other products $A_{1 \sigma(1)} A_{2 \sigma(2)} \cdots A_{d \sigma(d)}$, where $\sigma$ is a permutation of $\{1, \ldots, d\}$, are dominated in absolute value by $C_{1} s^{3}$, the determinant is non-zero for sufficiently small $s>0$. This completes the proof of the claim.

As there are the appropriate number of vectors, these vectors form a basis for $\mathfrak{g}_{n} \ominus \operatorname{sp}\left\{t_{1}\right\}$. Recall that $X_{\beta}^{F}, F E\left(2 e_{n}\right)$ and $F E e_{1}-e_{n}$ all belong to $\mathcal{N}_{X} \backslash\left\{F E e_{1}+\right.$ $\left.e_{n}: F=R, I\right\}$, hence

$$
s p\left\{A d(h) \mathcal{N}_{Y}, A d(\exp s M)\left(\mathcal{N}_{X} \backslash\left\{F E e_{1}+e_{n}\right\}\right)\right\}=\mathfrak{g}_{n} \ominus s p\left\{t_{1}\right\} .
$$

Finally, our familiar calculation shows

$$
A d(\exp s M)\left(I E e_{1}+e_{n}\right)=a_{s} I E e_{1}+e_{n}+s b_{s} t_{1}
$$

where $b_{s}$ converges to a non-zero constant. It follows that for small enough $s$,

$$
\operatorname{sp}\left\{\operatorname{Ad}(h)\left(\mathcal{N}_{Y}\right), \operatorname{Ad}(\exp s M)\left(\mathcal{N}_{X}\right)\right\}=\mathfrak{g}_{n},
$$

as we desired to show.

Case 2: Both $X$ and $Y$ are dominant $S U$ type.

First, assume the Lie algebra is type $B_{n}$ or $C_{n}$. According to [9, Thm. 8.2] both $\mu_{X}^{2}$ and $\mu_{Y}^{2}$ belong to $L^{2}$. Applying Holder's inequality we see that $\mu_{X} * \mu_{Y} \in L^{2}$. Being compactly supported, it follows that $\mu_{X} * \mu_{Y}$ is in $L^{1}$, and hence is a measure that is absolutely continuous with respect to Lebesgue measure. (Note that the same argument applies to the Lie algebra of type $D_{n}$ unless one of $X$ or $Y$ is of type $S U(n)$.)

However, we prefer to give an argument that is independent of $[\mathbf{9}$ as the techniques will then have more general application and such an argument will be needed in the case of type $D_{n}$, in any case. For this, in the case of type $B_{n}$, put

$$
\begin{aligned}
\Omega & =\left\{F E e_{1}, F E e_{1} \pm e_{j}: j \geq 2, F=R, I\right\} \text { and } \\
\Omega_{0} & =\left\{R E e_{1}, I E e_{1}\right\}
\end{aligned}
$$


while in the case of type $C_{n}$, put

$$
\begin{aligned}
\Omega & =\left\{F E\left(2 e_{1}\right), F E e_{1} \pm e_{j}: j \geq 2, F=R, I\right\} \text { and } \\
\Omega_{0} & =\left\{R E\left(2 e_{1}\right), I E\left(2 e_{1}\right)\right\} .
\end{aligned}
$$

In either case $\operatorname{ad}(H)(\Omega) \subseteq s p \Omega$ for all $H \in \mathfrak{g}_{n-1}$.

As $X, Y$ are dominant $S U$ type, both $\Omega_{X}$ and $\Omega_{Y}$ contain $F E(2) e_{1}$ and all the roots $F E e_{1}+e_{j}, j \geq 2$. If $g \in G_{n-1}$ is the Weyl conjugate that changes the signs of the letters $2, \ldots, n$, then $\left\{A d(g)\left(\Omega_{X}\right), \Omega_{Y} \backslash \Omega_{0}\right\}=\Omega$. Now take $M=R E(2) e_{1}$ and apply the general strategy.

The arguments are similar when the Lie algebra is type $D_{n}$. Let

$$
\Omega=\left\{F E e_{1} \pm e_{j}: j \geq 2, F=R, I\right\} .
$$

As we do not permit both $X$ and $Y$ to be of type $S U(n)$, without loss of generality $\Omega_{X}$ contains all the roots $F E e_{1}+e_{j}$ for $2 \leq j \leq n-1$, as well as both $F E e_{1} \pm e_{n}$, and $\Omega_{Y}$ contains either all $F E e_{1}+e_{j}$ for $2 \leq j$ or all $F E e_{1}+e_{j}$ for $2 \leq j \leq n-1$ and $F E e_{1}-e_{n}$. Let $\Omega_{0}$ be the choice of $\left\{F E e_{1}+e_{n}\right\}$ or $\left\{F E e_{1}-e_{n}\right\}$, depending on which belongs to $\Omega_{Y}$. Let $g \in G_{n-1}$ be the Weyl conjugate that changes the signs $2, \ldots, n-1$ (and $n$ if needed to be an even sign change). Then $\operatorname{Ad}(g)\left(\Omega_{X}\right) \supseteq$ $\left\{F E e_{1}-e_{j}, F E e_{1} \pm e_{n}: j \geq 2\right\}$ and hence $\left\{A d(g)\left(\Omega_{X}\right), \Omega_{Y} \backslash \Omega_{0}\right\}=\Omega$. Take $M=R E e_{1} \pm e_{n}$ with the choice of \pm depending on which belongs to $\Omega_{Y}$.

Case 3: $X$ and $Y$ are of different dominant type.

Without loss of generality assume $X$ is dominant $S U(m)$ type and $Y$ is dominant $B_{J}, C_{J}$ or $D_{J}$ type, depending on the type of the Lie algebra. Eligibility implies that $2 J+m \leq 2 n$.

Let

$$
\Omega=\left\{F E e_{1} \pm e_{j},\left(F E(2) e_{1}\right): j \geq 2\right\} .
$$

(with the inclusion of $F E e_{1}$ if the Lie algebra is type $B_{n}$ or $F E\left(2 e_{1}\right)$ if the Lie algebra is $C_{n}$ ). We have

$$
\Omega_{X}=\left\{F E e_{1}+e_{j}, F E e_{1}-e_{n}: j<n,\right\} \text { if } X=(a, \ldots, a,-a) \text { in } D_{n}
$$

and

$$
\Omega_{X}=\left\{F E e_{1}+e_{j}, F E e_{1}-e_{k},\left(F E(2) e_{1}\right): j \geq 2, k>m, F=R, I\right\} \text { otherwise. }
$$

Put

$$
\Omega_{0}=\left\{F E e_{1}+e_{n-J+1}\right\} \subseteq \Omega_{X} \cap \Omega_{Y}
$$

(or $\Omega_{0}=\left\{F E e_{1}-e_{n}\right\}$ if $J=1$ and $X=(a, \ldots, a,-a)$ in $\left.D_{n}\right)$. Applying a Weyl conjugate from $G_{n-1}$, we can assume

$$
\Omega_{Y}=\left\{F E e_{1} \pm e_{j}: 2 \leq j \leq n-J+1, F=R, I\right\} .
$$

If $n-J+1 \geq m$, then we already have

$$
\left\{\Omega_{Y}, \Omega_{X} \backslash \Omega_{0}\right\}=\Omega,
$$

so property (i) of the general strategy holds with $g=I d$. Take $M=R E e_{1}+e_{n-J+1}$ (resp., take $M=R E e_{1}-e_{n}$ ) to complete the argument.

Otherwise $m+J-n \geq 2$ (which implies $J \geq 2$ ). Put

$$
\Omega_{1}=\left\{F E e_{1}+e_{k}: 2 \leq k \leq n-J, F=R, I\right\} \subseteq\left(\Omega_{Y} \cap \Omega_{X}\right) \backslash \Omega_{0}
$$


and define

$$
H=\sum_{j=2}^{J-1} R E e_{j}+e_{n-J+j}+R E e_{J-} e_{n} \text { if } X=(a, \ldots, a,-a) \text { in type } D_{n}
$$

and

$$
H=\sum_{j=2}^{m+J-n} R E e_{j}+e_{n-J+j} \text { otherwise. }
$$

As $J \neq n, e_{j}+e_{n-J+j}$ are roots of the Lie algebra $\mathfrak{g}_{n-1}$. Let $2 \leq k \leq m+J-n$. Observe that $k \neq n-J+j$ for any $j \geq 2$, for if so, then $j=k-n+J \leq m+2 J-2 n$ and therefore the eligibility condition would imply $j \leq 0$. Thus, if $2 \leq k \leq m+J-n$, then $\operatorname{ad}(H)\left(F E e_{1}+e_{k}\right)=c_{k} F E e_{1}-e_{n-J+k}\left(\right.$ or $a d(H)\left(F E e_{1}+e_{J}\right)=c_{J} F E e_{1}+e_{n}$ if $X=(a, \ldots,-a))$.

The eligibility condition also implies

$$
\Omega_{1} \supseteq\left\{F E e_{1}+e_{k}: 2 \leq k \leq m+J-n\right\},
$$

therefore

$$
s p\left\{a d(H)\left(\Omega_{1}\right)\right\} \supseteq s p\left\{F E e_{1}-e_{j}, F E e_{1}+e_{n}: n-J+2 \leq j \leq n-1, F=R, I\right\}
$$

if $X=(a, \ldots, a,-a)$ in type $D_{n}$ and

$$
\operatorname{sp}\left\{a d(H)\left(\Omega_{1}\right)\right\} \supseteq \operatorname{sp}\left\{F E e_{1}-e_{j}: n-J+2 \leq j \leq m, F=R, I\right\} \text { otherwise. }
$$

Since $\Omega_{Y} \backslash \Omega_{1}=\left\{F E e_{1}-e_{j}, F E e_{1}+e_{n-J+1}: 2 \leq j \leq n-J+1\right\}$, in either case we have

$$
\operatorname{sp}\left\{\operatorname{ad}(H)\left(\Omega_{1}\right), \Omega_{Y} \backslash \Omega_{1}, \Omega_{X} \backslash \Omega_{0}\right\}=\operatorname{sp} \Omega .
$$

By Lemma 5 there is some $g \in G_{n-1}$ (namely, $g=\exp t H$ for sufficiently small $t$ ) such that

$$
\operatorname{sp}\left\{\operatorname{Ad}(g)\left(\Omega_{Y}\right), \Omega_{X} \backslash \Omega_{0}\right\}=\operatorname{sp} \Omega .
$$

Again, take $M=R E e_{1}+e_{n-J+1}$ and apply the general strategy to complete the argument.

Part II: $g_{n}$ is type $S U(n)$.

This is very similar to case $1(\mathrm{a})$. Let

$$
\Omega=\left\{F E e_{1}-e_{j}: 2<j \leq n, F=R, I\right\} .
$$

We have

$$
\begin{aligned}
& \Omega_{X}=\left\{F E e_{1}-e_{j}: S_{X}<j \leq n, F=R, I\right\} \text { and } \\
& \Omega_{Y}=\left\{F E e_{1}-e_{j}: S_{Y}<j \leq n, F=R, I\right\} .
\end{aligned}
$$

Put $\Omega_{0}=\left\{F E e_{1}-e_{n}: F=R, I\right\}$. Take $g \in S U(n-1)$ to be the Weyl conjugate that interchanges the letters $S_{Y}+j$ and $1+j$ for $j=1, \ldots, S_{X}-1$. The eligibility condition ensures this is well defined and leaves 1 and $n$ unchanged. Clearly $\left\{A d(g)\left(\Omega_{Y}\right), \Omega_{X} \backslash \Omega_{0}\right\}=\Omega$. Take $M=R E e_{1}-e_{n}$ and apply the general strategy in the usual manner. 


\section{Proof of the Main Theorem}

In this section we will complete the proof of Theorem 1

Proof of Theorem 1, Necessary conditions for Absolute continuity: Lemma 1 shows that absolutely continuous tuples are eligible, while in Lemma 2 we saw that the exceptional tuples, other than possibly the pairs of type $(S U(n), S U(n-$ 1)) in the Lie algebra of type $D_{n}$ with $n \geq 6$, are not absolutely continuous.

The rest of the proof is devoted to establishing that the eligible, non-exceptional tuples are absolutely continuous.

Sufficient conditions for Absolute continuity for Lie types $A_{n}, B_{n}$ and $C_{n}$ :

Case $L=2$. The proof proceeds by induction on the rank $n$ of the Lie algebra. We begin $A_{n}$ with $n=1$ (type $\left.S U(2)\right)$ and $B_{n}$ with $n=2$. Although it is customary to only define $C_{n}$ for $n \geq 3$, there is no harm in beginning with $C_{2}$, meaning the root system $\pm\left\{2 e_{1}, 2 e_{2}, e_{1} \pm e_{2}\right\}$, which is Lie isomorphic to $B_{2}$.

According to [9. Thm. 8.2], all non-zero pairs $(X, Y)$ in the Lie algebras of type $S U(2)$ and $B_{2}$ have the property that both $\mu_{X}^{2}, \mu_{Y}^{2} \in L^{2}$. Thus $\mu_{X} * \mu_{Y}$ is a compactly supported measure in $L^{2}$ and hence is an absolutely continuous measure. The existence of $g_{1}, g_{2} \in G_{n}$ with

$$
\sum_{i=1}^{2} T_{A d\left(g_{i}\right)\left(X_{i}\right)}\left(O_{X_{i}}\right)=\mathfrak{g}_{n}
$$

is a Lie isomorphism invariant, thus from Prop. 1 we can also deduce that $\mu_{X} * \mu_{Y}$ is an absolutely continuous measure for all non-zero $\left(X_{1}, X_{2}\right)$ in the Lie algebra of type $C_{2}$.

Now, inductively assume that all eligible, non-exceptional pairs in $S U(n-1)$, $B_{n-1}$ or $C_{n-1}$, with $n \geq 3$, are absolutely continuous. (Of course, there are no exceptional pairs in $B_{n-1}$ or $C_{n-1}$.)

Let $(X, Y)$ be an eligible, non-exceptional pair in $S U(n), B_{n}$ or $C_{n}$, and form the reduced pair $\left(X^{\prime}, Y^{\prime}\right)$. The reduced pair is eligible by Lemma 3 , Notice that only an element of type $S U\left(\frac{n+1}{2}\right) \times S U\left(\frac{n-1}{2}\right)$ in $S U(n)$ will reduce to an element of type $S U\left(\frac{n-1}{2}\right) \times S U\left(\frac{n-1}{2}\right)$ in $S U(n-1)$. Furthermore, a pair of elements each of type $S U\left(\frac{n+1}{2}\right) \times S U\left(\frac{n-1}{2}\right)$ is not eligible in $S U(n)$, thus we can assume $\left(X^{\prime}, Y^{\prime}\right)$ is both eligible and non-exceptional. By the induction assumption, $\left(X^{\prime}, Y^{\prime}\right)$ is an absolutely continuous pair. But then the induction step, Prop. 3 implies that $(X, Y)$ is absolutely continuous.

Case $L \geq 3$. Again, we proceed by induction on $n$. We remark that as $\mu * \nu$ is absolutely continuous if $\mu$ is absolutely continuous and $\nu$ is an arbitrary measure, the fact that the convolution of any two non-zero orbital measures in type $S U(2), B_{2}$ or $C_{2}$ is absolutely continuous, proves that the same is true for the convolution of any $L$ non-zero orbital measures. This starts the induction.

First, suppose $\left(X_{1}, \ldots, X_{L}\right)$ is an eligible $L$-tuple in $B_{n}$ or $C_{n}$ with $n \geq 3$. We will let $\Omega$ be as in Prop. 3, depending on whether $\mathfrak{g}$ is type $B_{n}$ or $C_{n}$,

$$
\Omega=\left\{F E e_{1} \pm e_{j}, F E(2) e_{1}: j=2, \ldots, n, F=R, I\right\} .
$$

As a pair of elements that is dominant $S U$ type in $B_{n}$ or $C_{n}$ is eligible and not exceptional, the theorem for $L=2$ implies the convolution of (even) their two 
orbital measures is absolutely continuous. Thus we may assume that at most one $X_{i}$ is dominant $S U$ type.

Suppose that no $X_{i}$ are dominant $S U$ type and form the corresponding $X_{i}^{\prime}$. If $X_{i}^{\prime}$ and $X_{j}^{\prime}$ are dominant $S U$, then the pair $\left(X_{i}, X_{j}\right)$ is eligible (and not exceptional), thus $\mu_{X_{i}} * \mu_{X_{j}}$ is absolutely continuous. Hence we can assume that at most one $X_{i}^{\prime}$ is dominant $S U$ type.

Since $S_{X^{\prime}}=S_{X}-2$ when both $X$ and $X^{\prime}$ are dominant $B$ (or $C$ ) type it follows that

$$
\sum_{i=1}^{L} S_{X_{i}^{\prime}} \leq \sum_{i=1}^{L} S_{X_{i}}-2(L-1) \leq 2 n(L-1)-2(L-1)=2(n-1)(L-1) .
$$

This shows that $\left(X_{1}^{\prime}, \ldots, X_{L}^{\prime}\right)$ is eligible in $\mathfrak{g}_{n-1}$. As it is not exceptional, the induction assumption implies it is an absolutely continuous tuple.

Here $\Omega_{X_{i}}=\left\{F E e_{1} \pm e_{j}: j>J_{i}\right\}$ where $2 J_{i}=S_{X_{i}}$. Taking $g_{i}$ to be the Weyl conjugate that switches appropriate letters (and fixes the letters 1 and $n$ ) we can arrange for

$$
A d\left(g_{i}\right)\left(\Omega_{X_{i}}\right)=\left\{F E e_{1} \pm e_{j}: j=(i-1) n-\sum_{k=1}^{i-1} J_{k}+2, \ldots, \text { in }-\sum_{k=1}^{i} J_{k}+1\right\}
$$

(with suitable modifications if any of the specified choices of $j$ exceed $n$ ).

If $(L-1) n-\sum_{k=1}^{L-1} J_{k}+1 \geq n$, then

$$
\bigcup_{i=1}^{L-1} A d\left(g_{i}\right)\left(\Omega_{X_{i}}\right)=\left\{F E e_{1} \pm e_{j}: j=2, \ldots, n\right\},
$$

and this coincides with the set $\Omega_{Y}$ for a suitable $Y$ of type $B_{1}$ (or $C_{1}$ ) (meaning type $B_{1}\left(\right.$ or $\left.\left.C_{1}\right) \times S U(1) \times \cdots \times S U(1)\right)$. As always $S_{X} \leq 2(n-1)$, the pair $\left(Y, X_{L}\right)$ is eligible.

Otherwise, if we let $m=n-(L-1) n+\sum_{i=1}^{L-1} J_{i}$ and take a suitable choice of $Y$ of type $B_{m}$ (or $\left.C_{m}\right)$, then

$$
\bigcup_{i=1}^{L-1} A d\left(g_{i}\right) \Omega_{X_{i}}=\Omega_{Y}
$$

The eligibility condition ensures that

$$
\begin{aligned}
S_{Y}+S_{X_{L}} & =2\left(n-(L-1) n+\sum_{i=1}^{L-1} J_{i}\right)+2 J_{L} \\
& \leq 2 n-2(L-1) n+\sum_{i=1}^{L} S_{X_{i}} \leq 2 n
\end{aligned}
$$

and thus the pair $\left(Y, X_{L}\right)$ is eligible and clearly not exceptional. The arguments given in the proof of Prop. 3. Case 1 show that then there is some $g \in G_{n-1}, M \in \mathfrak{g}_{n}$ and $\Omega_{0} \subseteq \Omega_{X_{L}}$ such that

(i) $\operatorname{sp} \Omega=\operatorname{sp}\left\{\operatorname{Ad}(g)\left(\Omega_{Y}\right), \Omega_{X_{L}} \backslash \Omega_{0}\right\}$;

(ii) $a d^{k}(M): \mathcal{N}_{X_{L}} \backslash \Omega_{0} \rightarrow \operatorname{sp}\left\{\Omega, \mathfrak{g}_{n-1}\right\}$ for all positive integers $k$; and

(iii) The span of the projection of $\operatorname{Ad}(\exp s M)\left(\Omega_{0}\right)$ onto the orthogonal complement of $s p\left\{\mathfrak{g}_{n-1}, \Omega\right\}$ in $\mathfrak{g}_{n}$ is a surjection for all small $s>0$. 
Since

$$
\operatorname{sp}\left\{A d(g)\left(\Omega_{Y}\right), \Omega_{X_{L}} \backslash \Omega_{0}\right\} \subseteq \operatorname{sp}\left\{A d\left(g g_{i}\right)\left(\Omega_{X_{i}}\right), \Omega_{X_{L}} \backslash \Omega_{0}: i=1, \ldots, L-1\right\}
$$

we can call upon the general strategy, Prop. 2, with $g_{i}$ replaced there by $g g_{i}$, to deduce that $\left(X_{1}, \ldots, X_{L}\right)$ is an absolutely continuous tuple. This completes the argument when no $X_{i}$ are of dominant $S U$ type.

Otherwise, there is one $X_{i}$ which is of dominant $S U$ type, say $X_{L}$. If there is another index $j$ such that $X_{j}^{\prime}$ is of dominant $S U$ type, then the pair $\left(X_{L}, X_{j}\right)$ is eligible and not exceptional, hence $\mu_{X_{L}} * \mu_{X_{j}}$ is absolutely continuous.

So we may assume all $X_{j}^{\prime}$ with $j \neq L$ are of dominant $B$ (or $C$ ) type. Thus

$$
\sum S_{X_{i}}^{\prime} \leq \sum S_{X_{i}}-2(L-1) \leq 2(n-1)(L-1),
$$

so $\left(X_{1}^{\prime}, \ldots, X_{L}^{\prime}\right)$ is an eligible $L$-tuple. Again, taking $g_{i}$ to be suitable Weyl conjugates we have

$$
\bigcup_{i=1}^{L-1} A d\left(g_{i}\right)\left(\Omega_{X_{i}}\right)=\left\{F E e_{1} \pm e_{j}: j=2, \ldots,(L-1) n-\sum_{i=1}^{L-1} J_{i}+1\right\}
$$

and if we let $Y$ be of type $B_{m}$ where $m=n-(L-1) n+\sum_{i=1}^{L-1} J_{i}$, then

$$
\bigcup_{i=1}^{L-1} A d\left(g_{i}\right)\left(\Omega_{X_{i}}\right)=\Omega_{Y}
$$

The eligibility condition ensures that

$$
S_{Y}+S_{X_{L}}=2\left(n-(L-1) n+\sum_{i=1}^{L-1} J_{i}\right)+S_{X_{L}} \leq 2 n
$$

so the pair $\left(Y, X_{L}\right)$ is eligible. Complete the proof using the arguments of Prop. 3 . but this time using Case 3 as $X_{L}$ and $Y$ are of opposite dominant types.

The argument is similar, but easier, if the Lie algebra is type $S U(n)$. We first check that $\left(X_{1}^{\prime}, \ldots, X_{L}^{\prime}\right)$ is eligible when $\left(X_{1}, \ldots, X_{L}\right)$ is eligible. This is clear if at most one $X_{i}$ has $S_{X_{i}}=S_{X_{i}^{\prime}}$. If two or more $X_{i}$ have $S_{X_{i}}=S_{X_{i}^{\prime}}$, then these two satisfy $S_{X_{i}} \leq n / 2$ and because all $S_{X^{\prime}} \leq n-2$, we have

$$
\sum_{i=1}^{L} S_{X_{i}^{\prime}} \leq 2(n / 2)+(L-2)(n-2) \leq(L-1)(n-1)
$$

proving eligibility.

Set $\Omega=\left\{F E e_{1}-e_{j}: 2 \leq j \leq n\right\}$. We have $\Omega_{X_{i}}=\left\{F E e_{1}-e_{j}: j>S_{X_{i}}\right\}$. Upon taking $g_{i}$ suitable Weyl conjugates that permute letters we obtain

$$
\bigcup_{i=1}^{L-1} A d\left(g_{i}\right) \Omega_{X_{i}}=\Omega_{Y}
$$

where $Y$ is an element of the torus of $S U(n)$ of type $S U(m)$ with $m=n-(L-$ 1) $n+\sum_{i=1}^{L-1} J_{i}$. The eligibility assumption ensures $\left(X_{L}, Y\right)$ is an eligible pair and it is clearly not exceptional. Now complete the argument using the $L=2$ case in the same manner as for type $B_{n}$ and $C_{n}$. 
The many exceptional pairs in $D_{n},(n=4$ in particular $)$, cause complications in proving the theorem for type $D_{n}$. We will again prove the main theorem by an induction argument for $L=2$, but it will be convenient to begin the argument with type $D_{5}$. In the next lemma we will prove that all eligible, non-exceptional pairs in $D_{4}$ and $D_{5}$ are absolutely continuous. This will start the base case for us.

We will actually begin with $D_{3}$. Usually $D_{n}$ is defined for $n \geq 4$, but that is because $D_{3}$ is Lie isomorphic to type $A_{3}$. As the problem of characterizing the $L$ tuples in type $A_{3}$ has already been done we can use this characterization, together with the induction step, Prop. 3. to handle most of the eligible, non-exceptional pairs in $D_{4}$ and $D_{5}$. This approach will work whenever the reduced pair is known to be an absolutely continuous pair (in $D_{3}$ or $D_{4}$, respectively). There will still be a few remaining pairs to consider and these will be handled directly by verifying Wright's criteria for absolute continuity, Thm. 2.

Lemma 6. All the eligible, non-exceptional pairs in $D_{4}$ and $D_{5}$ are absolutely continuous.

Proof. As explained above, we begin the proof by considering $D_{3}$. Under the Lie isomorphism between $D_{3}$ and $A_{3}$, any subsystem of type $D_{2}$ in $D_{3}$ is isomorphic to one of type $A_{1} \times A_{1}$, type $D_{1}$ is isomorphic to one of type $A_{0}$ (or $S U(1)$ ) and types $S U(j)$ for $j=1,2,3$ are unchanged under such an isomorphism. With this observation and the criteria for absolute continuity already known for the Lie algebra of type $A_{3}$, it is easy to check that all pairs $(X, Y)$ in $D_{3}$ are absolutely continuous except those of type $\left(D_{2}, D_{2}\right),\left(D_{2}, S U(3)\right),(S U(3), S U(3))$ and $(S U(3), S U(2))$, the first two of these being not eligible and the latter two, exceptional.

Case $D_{4}$ : Prop. 3 guarantees that all eligible, non-exceptional pairs, $(X, Y)$, in $D_{4}$ are absolutely continuous, except when the reduced pair, $\left(X^{\prime}, Y^{\prime}\right)$, is one of the four pairs listed above. Furthermore, because we have already seen that the pair $\left(X^{\prime}, Y^{\prime}\right)$ is eligible whenever $(X, Y)$ is an eligible, non-exceptional pair, we will only need to give a special argument for those pairs $(X, Y)$ where $X^{\prime}$ is type $S U(3)$ and $Y^{\prime}$ is either type $S U(3)$ or $S U(2)$ (the latter being type $S U(2) \times D_{1}$ or $S U(2) \times S U(1))$.

Thus we are left to study the pairs $(X, Y)$ where $X$ is of type $S U(4)$ and $Y$ is one of type $S U(4)$, type $S U(3)$ (to be more precise, either type $S U(3) \times D_{1}$ or $S U(3) \times S U(1))$, type $S U(2) \times D_{2}$ or $S U(2) \times S U(2)$. However, these are all exceptional pairs except when $X$ is type $S U(4), Y$ is of type $S U(2) \times S U(2)$ and $\Phi_{Y}$ is not Weyl conjugate to a subset of $\Phi_{X}$.

To prove this last pair is absolutely continuous, we verify the criteria of Thm. 2 (with $X_{1}=X$ and $X_{2}=Y$ ) and follow the notation there. Here we have $|\Phi|=24$ and $\left|\Phi_{X_{1}}\right|+\left|\Phi_{X_{2}}\right|=12+4=16$. The rank 3, root subsystems, $\Psi$ of $D_{4}$, are those of type $D_{3}, S U(4)$ (two non-Weyl conjugate subsystems) and $D_{2} \times S U(2)$.

When $\Psi$ is type $D_{2} \times S U(2)$, then $|\Psi|=6$. Thus we even have $|\Phi|-|\Psi|-1 \geq$ $\left|\Phi_{X_{1}}\right|+\left|\Phi_{X_{2}}\right|$, so (3.3) clearly holds. When $\Psi$ is type $D_{3}$, then $|\Psi|=12$. However, $\left|\Phi_{X_{1}} \cap \sigma(\Psi)\right|=6$ and $\left|\Phi_{X_{2}} \cap \sigma(\Psi)\right| \geq 2$ for all choices of $\sigma \in W$ because $\sigma(\Psi)$ must contain $\pm e_{i} \pm e_{j}, \pm e_{i} \pm e_{k}, \pm e_{j} \pm e_{k}$ for three choices of letters $i, j, k$. Thus the LHS of (3.3) is 11 , while the RHS is at most 8.

Now assume $\Psi$ is type $S U(4)$. First, suppose $\Psi$ is Weyl conjugate to the set of annihilators of $X$. Since we need to calculate the intersection of $\Phi_{X_{j}}$ with all Weyl conjugates of $\Psi$, there is no loss of generality in assuming $\Phi_{X_{1}}=\Psi=\left\{e_{i}-e_{j}: 1 \leq\right.$ 
$i \neq j \leq 4\}$. By assumption, $\Phi_{X_{2}}$ is not Weyl conjugate to a subset of $\Phi_{X_{1}}$, thus there is also no loss of generality in assuming $\Phi_{X_{2}}=\left\{ \pm\left(e_{1}-e_{2}\right), \pm\left(e_{3}+e_{4}\right)\right\}$.

The reader can check that $\left|\Phi_{X_{1}} \cap \sigma(\Psi)\right|$ is minimal when we take the choice of $\sigma \in W$ that switches two signs and in this case $\left|\Phi_{X_{1}} \cap \sigma(\Psi)\right|=4$. Similarly, it can be shown that if $\sigma$ is any Weyl conjugate, then $\left|\Phi_{X_{2}} \cap \sigma(\Psi)\right| \geq 2$, so that again the LHS of (3.3) is 11 and the RHS is at most 10.

Finally, suppose $\Psi$ is not Weyl conjugate to $\Phi_{X_{1}}$. Without loss of generality we can assume $\Psi$ is as before and $\Phi_{X_{1}}=\left\{e_{i}-e_{j}, \pm\left(e_{4}+e_{j}\right): 1 \leq i \neq j \leq 3\right\}$. Again $\left|\Phi_{X_{1}} \cap \sigma(\Psi)\right|$ is minimal when $\sigma$ is the Weyl element that switches two signs, but in this case $\left|\Phi_{X_{1}} \cap \sigma(\Psi)\right|=6$. This is already enough to establish (3.3) and completes the argument that $(X, Y)$ is an absolutely continuous pair.

Case $D_{5}$ : Again, Prop. 3 implies we only need to study the eligible nonexceptional pairs, $(X, Y)$ in $D_{5}$, where the reduced pair has $X^{\prime}$ of type $S U(4)$ and $Y^{\prime}$ one of type $S U(4), S U(3), S U(2) \times D_{2}$, or $S U(2) \times S U(2)$. Since the pairs $\left(S U(5), S U(5)\right.$ and $(S U(5), S U(4))$ are exceptional and the pair $\left(S U(5), D_{3} \times\right.$ $S U(2))$ is not eligible, this reduces the problem to the study of the pairs $(X, Y)$ where $X$ is of type $S U(5)$ and $Y$ is either of type $S U(3) \times D_{2}$ or $S U(3) \times S U(2)$. Further, since the set of annihilators of an element of type $S U(3) \times S U(2)$ is contained in the set of annihilators of an element of type $S U(3) \times D_{2}$, it will suffice to prove that the pair $\left(S U(5), S U(3) \times D_{2}\right)$ is absolutely continuous.

For this, we again use Thm. 2 In $D_{5}$, the rank 4 root subsystems $\Psi$ which we must study are those of type $D_{4}, S U(5), D_{3} \times S U(2)$ and $D_{2} \times S U(3)$, with cardinalities 24,20,14 and 10, respectively. The cardinality of $\Phi$ is 40 , while $\left|\Phi_{X_{1}}\right|=20$ and $\left|\Phi_{X_{2}}\right|=10$.

Let $\Lambda$ be a root subsystem of type $D_{2}, D_{3}$ or $D_{4}$ in $D_{5}$. It is easy to see that if $\Lambda$ is a root subsystem of type $D_{j}$ in $D_{5}$, with $j=2,3,4$, then $\left|\Phi_{X_{1}} \cap \Lambda\right|=\frac{1}{2}|\Lambda|$. Furthermore, $\left|\Phi_{X_{2}} \cap \Lambda\right|=6$ whenever $\Lambda$ is type $D_{4}$. Since the action of a Weyl element preserves the type of a root subsystem, these calculations can be used to show that (3.3) holds if $\Psi$ is type $D_{4}, D_{3} \times S U(2)$ or $D_{2} \times S U(3)$.

When $\Psi$ is type $S U(5)$, then $\left|\Phi_{X_{1}} \cap \sigma(\Psi)\right| \geq 8$ for all $\sigma$ (with the minimum occurring when $\sigma$ is two sign changes). Moreover, $\left|\Phi_{X_{2}} \cap \sigma(\Psi)\right| \geq 4$ so that again (3.3) is satisfied. This shows that the pair $\left(S U(5), S U(3) \times D_{2}\right)$ is absolutely continuous and completes the base case arguments.

Further complications arise with type $D_{n}$ because of the fact that when $X$ is of type $S U(n)$, then $\mu_{X}^{2}$ is not absolutely continuous. We have already seen this complication in the proof of Prop. 3 (when $L=2$ ), but it presents further difficulties when $L>2$. To handle this, we introduce the following terminology for the remainder of the proof.

Definition 5 . We will say that $X$ is almost dominant $S U$ type if $X$ is type $D_{J} \times S U\left(s_{1}\right) \times \cdots \times S U\left(s_{t}\right)$ where $J \leq \sum s_{i}$.

Of course, if $X$ is dominant $S U$ type, then it is almost dominant $S U$ type. However, $X$ is also almost dominant $S U$ type if $X$ is dominant $D$ type, but $X^{\prime}$ is dominant $S U$ type, for instance. If $X$ is almost dominant $S U$ type and not type $S U(n)$, then [9, Thm. 8.2], implies $\mu_{X}^{2} \in L^{2}$. Here are some additional properties.

Lemma 7. Suppose $X_{1}, X_{2}$ are almost dominant $S U$ type in $D_{n}$ and $X_{3} \neq 0$.

(i) If neither $X_{1}$ nor $X_{2}$ are type $S U(n)$, then $\mu_{X_{1}} * \mu_{X_{2}} \in L^{2}$.

(ii) If $X_{1}$ and $X_{2}$ are both type $S U(n)$ and $X_{3}$ is not, then $\mu_{X_{1}} * \mu_{X_{2}} * \mu_{X_{3}} \in L^{2}$. 

$L^{2}$.

(iii) More generally, if $X_{1}$ is type $S U(n)$ and $X_{2}$ is not, then $\mu_{X_{1}} * \mu_{X_{2}} * \mu_{X_{3}} \in$

(iv) If $n \geq 5$ (or $n=4$ ) and $X_{3}$ (and $\left.X_{4}\right)$ is almost dominant $S U(n)$ type, then $\mu_{X_{1}} * \mu_{X_{2}} * \mu_{X_{3}}\left(* \mu_{X_{4}}\right) \in L^{2}$.

Proof. (i) follows from [9] as remarked above. The fact that it is absolutely continuous, which is actually all we will need for our application, also follows from the $L=2$ part of the proof of the main theorem since $\left(X_{1}, X_{2}\right)$ is an eligible, non-exceptional pair.

(iv) holds similarly from $\left[9\right.$ since $\mu_{X}^{3} \in L^{2}$ whenever $X$ is almost dominate $S U$ type and $n \geq 5$, and $\mu_{X}^{4} \in L^{2}$ when $n=4$. (Alternatively, absolute continuity can be checked from Theorem 2.)

For (ii) and (iii) we proceed by induction on $n$, noting that according to the main theorem, as already established for all $L \geq 2$ in the Lie algebras of type $A_{n}$, all triples in $A_{3}$ (equivalently, $D_{3}$ ) are absolutely continuous, except when all three are type $S U(3)$.

Now assume $n \geq 4$. We put

$$
\Omega=\left\{F E e_{1} \pm e_{j}: j=2, \ldots, n, F=R, I\right\} .
$$

(ii): Here $X_{1}^{\prime}, X_{2}^{\prime}$ will be of type $S U(n-1)$ in $D_{n-1}$, while $X_{3}^{\prime}$ is not, so the induction hypothesis applies. Without loss of generality we can assume

$$
\Omega_{X_{1}}=\left\{F E e_{1}+e_{j}: j \geq 2, F=R, I\right\}
$$

and $\Omega_{X_{2}}$ either coincides with $\Omega_{X_{1}}$ or

$$
\Omega_{X_{2}}=\left\{F E e_{1}+e_{j}, F E e_{1}-e_{n}: j \leq n-1, F=R, I\right\} .
$$

As $X_{3}$ is not of type $S U(n), \Omega_{X_{3}}$ contains $\left\{F E e_{1} \pm e_{n}\right\}$. Let $g$ be the Weyl conjugate changing the signs of $2, \ldots, n-1$ (and $n$ if needed to be an even sign change). Then $\Omega_{X_{1}} \cup A d(g)\left(\Omega_{X_{2}}\right)$ contains all of $\Omega$ except for possibly $\left\{F E e_{1}-e_{n}\right.$ : $F=R, I\}$. If $\Omega_{0}=\left\{F E e_{1}+e_{n}\right\}$, we have

$$
\Omega_{X_{1}} \cup \operatorname{Ad}(g)\left(\Omega_{X_{2}}\right) \cup\left(\Omega_{X_{3}} \backslash \Omega_{0}\right)=\Omega .
$$

Taking $M=R E e_{1}+e_{n}$ one can verify that the hypotheses of the general strategy, Prop. 2, are all satisfied. Consequently, $\left(X_{1}, X_{2}, X_{3}\right)$ is absolutely continuous.

(iii): We define $X_{1}^{\prime}$ and $X_{3}^{\prime}$ as usual, but will re-define $X_{2}^{\prime}$ so that it continues to be of almost dominant $S U$ type and not type $S U(n-1)$ (so that we will be able to apply the induction hypothesis). This can be achieved by defining $X_{2}^{\prime}$ to be type $D_{J-1} \times S U\left(s_{1}\right) \times \cdots \times S U\left(s_{t}\right)$ if $X_{2}$ is type $D_{J} \times S U\left(s_{1}\right) \times \cdots \times S U\left(s_{t}\right)$ with $J>1$, or defining $X_{2}^{\prime}$ to be type $D_{J} \times S U\left(s_{1-1}\right) \times \cdots \times S U\left(s_{t}\right)$ if $J=0$ or 1 and $s_{1}=\max s_{j}$. The fact that $X_{2}$ is almost dominant $S U$ type ensures that $J \leq n / 2$, so whether $X_{2}$ is dominant $S U$ type or not, $S_{X_{2}} \leq n$ and thus $\left(X_{1}, X_{2}\right)$ is an eligible pair. Further, $X_{1}, X_{2}$ are not both of type $S U(n)$.

The arguments given in Prop. 3, Case 2 or 3 depending on the situation, can be applied to prove there is some $g \in D_{n-1}$ such that $\operatorname{sp}\left\{\Omega_{X_{1}} \backslash \Omega_{0}, A d(g)\left(\Omega_{X_{2}}\right)\right\}=s p \Omega$ where $\Omega_{0}$ is taken to be the choice of $F E e_{1}+e_{n}$ or $F E e_{1}-e_{n}$ that belongs to $\Omega_{X_{1}}$. Therefore

$$
\operatorname{sp}\left\{\Omega_{X_{1}} \backslash \Omega_{0}, A d(g)\left(\Omega_{X_{2}}\right), \Omega_{X_{3}}\right\}=s p \Omega .
$$

Now take $M=R E e_{1} \pm e_{n}$ (depending on the choice of $\Omega_{0}$ ) and apply the general strategy. 
We are now ready to conclude the proof of Theorem 1 by completing the proof of sufficiency for absolute continuity in type $D_{n}$.

Proof of Theorem 1 continued. Sufficient conditions for Absolute continuity for Lie type $D_{n}$ :

Case $L=2$. Lemma 6 starts the induction argument for type $D_{n}$. Now, inductively assume that all eligible, non-exceptional pairs in $D_{n-1}$, with $n \geq 6$, are absolutely continuous. By Lemma 3 the pair $\left(X^{\prime}, Y^{\prime}\right)$ is eligible. If it is an exceptional pair, then it must be either of type $(S U(n-1), S U(n-1))$ or type $(S U(n-1), S U(n-2))$ (where the $S U(n-2)$ could be type $S U(n-2) \times D_{1}$ or $S U(n-2) \times S U(1))$. But then $(X, Y)$ must also have been an exceptional pair in $D_{n}$, which is a contradiction. By the induction assumption, $\left(X^{\prime}, Y^{\prime}\right)$ is an absolutely continuous pair and hence Prop. 3 implies that $(X, Y)$ is absolutely continuous.

Case $L \geq 3$. Again, we give an induction argument. The base case, $D_{4}$, will be discussed at the conclusion of the proof. So assume $n \geq 5$ and $\left(X_{1}, \ldots, X_{L}\right)$ is an eligible $L$-tuple in $D_{n}$. We note that there are no exceptional $L$-tuples in $D_{n}$ for $n \geq 5$ when $L \geq 3$.

We will take

$$
\Omega=\left\{F E e_{1} \pm e_{j}: j=2, \ldots, n\right\} .
$$

More care is needed in this situation then for the Lie algebras of type $B_{n}$ and $C_{n}$, since the fact that $\mu_{X}^{2} \notin L^{2}$ when $X$ is of type $S U(n)$ means, for example, that we cannot immediately assume that at most one $X_{i}$ is dominant $S U$ type, as we did in the argument for those Lie types. Here is where Lemma 7 will be useful.

If three or more $X_{i}$ are dominant $S U$ type, then the induction argument is not even necessary as Lemma 7 (iv) implies that their convolution is already in $L^{2}$ and hence is absolutely continuous.

If two $X_{i}$ (say, $X_{1}, X_{2}$ ) are both dominant $S U$ type and some $X_{j}$, say $X_{3}$, is not, then we call upon one of the first three parts of the lemma.

So we can assume there is at most one $X_{i}$ that is dominant $S U$ type, say $X_{1}$. If there is some $X_{j}$, other than $X_{1}$, with $X_{j}^{\prime}$ of dominant $S U$ type, then $X_{j}$ is almost dominant $S U$ and not type $S U(n)$. Apply the appropriate part of Lemma 7 with $X_{1}, X_{2}$ equal to this $X_{j}$, and $X_{3}$ any other $X_{i}$.

If all $X_{j}^{\prime}$, other than $j=1$, remain dominant $D$ type, then the calculations used in the type $B_{n}$ or $C_{n}$ case show that $\left(X_{1}^{\prime}, \ldots, X_{L}^{\prime}\right)$ is an eligible, non-exceptional tuple in $D_{n-1}$. For the induction step we argue in the same fashion as we did for the Lie algebras of type $B_{n}$ or $C_{n}$ in the same situation.

Finally, assume all $X_{j}$ are dominant $D$ type. If two or more $X_{j}^{\prime}$ are dominant $S U$ type, then the corresponding two $X_{j}$ are almost dominant $S U$ type and not of type $S U(n)$. Their convolution is even in $L^{2}$. If at most one $X_{j}^{\prime}$ is dominant $S U$ type, then $\left(X_{1}^{\prime}, \ldots, X_{L}^{\prime}\right)$ is an eligible tuple, so the induction hypothesis applies. The induction step is the same as for the corresponding situation with type $B_{n}$ or $C_{n}$.

To conclude, we must establish the base case, $n=4$. Since $\mu_{X}^{4} \in L^{2}$ for any non-trivial $X$ in the Lie algebra of type $D_{4}$, every $L$-tuple with $L \geq 4$ is an absolutely continuous tuple.

So we can assume $L=3$. The induction argument above can be applied to $\left(X_{1}, X_{2}, X_{3}\right)$ provided at most one $X_{j}$ is dominant $S U$ type, hence for such triples it suffices to check that $\left(X_{1}^{\prime}, X_{2}^{\prime}, X_{3}^{\prime}\right)$ in $D_{3}$ is an absolutely continuous triple. But 
this follows from the main theorem for type $A_{n}$, since all triples in $A_{3}$, except when all $X_{i}$ are type $S U(3)$, are absolutely continuous.

If two or three $X_{j}$ are dominant $S U$ type, but at least one $X_{i}$ is not of type $S U(4)$, Lemma 7 gives the result.

If all three $X_{i}$ are type $S U(4)$ and their annihilating root systems are Weyl conjugate, then the triple, $\left(X_{1}, X_{2}, X_{3}\right)$, is exceptional. Thus we can assume the annihilating root systems are not Weyl conjugate. As the arguments are symmetric, there is no loss of generality in assuming that the set of annihilating roots for $X_{1}$ coincides with that of $X_{2}$ and is given by

$$
\Phi_{X_{1}}=\Phi_{X_{2}}=\left\{e_{i}-e_{j}: 1 \leq i \neq j \leq 4\right\},
$$

while

$$
\Phi_{X_{3}}=\left\{e_{i}-e_{j}, \pm\left(e_{4}+e_{k}\right): 1 \leq i \neq j \leq 3, k=1,2,3\right\} .
$$

We will again call upon Thm. 2 to check the absolute continuity of the triple. The root systems $\Psi$ of rank 3 that we must consider are those of type $D_{3}, S U$ (4) (two non-Weyl conjugate root subsystems) and $D_{2} \times S U(2)$.

Of course, $\sum_{i=1}^{3}\left|\Phi_{X_{i}}\right|=36$ and $|\Phi|=24$ when $\Phi$ is the root system of $D_{4}$. When $\Psi$ is type $D_{2} \times S U(2)$, then $|\Psi|=6$, and as $\Psi$ intersects non-trivially any root subsystem of type $S U(4)$, the inequality (3.3) is clear. When $\Psi$ is type $D_{3}$ it is easy to see that $\left|\Phi_{X_{i}} \cap \sigma(\Psi)\right| \geq 6$ for each $i$ and any Weyl element $\sigma$.

When $\Psi$ is type $S U(4)$, then $\left|\Phi_{X_{i}} \cap \sigma(\Psi)\right| \geq 4$. However, as noted in the $L=2$ argument, if $\Psi$ and $\Phi_{X_{i}}$ are non-Weyl conjugate subsystems of type $S U(4)$, then this lower bound can be improved to $\dot{6}$. Consequently, $2(|\Phi|-|\Psi|)-1=23$, while the right hand side of (3.3) is at most $36-(4+4+6)=22$, so the inequality holds.

This completes the base case argument and hence the proof of Theorem 1 .

\section{Applications}

7.1. Consequences of the Main Theorem. An element $X \in \mathfrak{t}_{n}$ is said to be regular if its set of annihilating roots is empty. These would be the elements of type $S U(1) \times \cdots \times S U(1)$ (in any Lie algebra) or $D_{1} \times S U(1) \times \cdots \times S U(1)$ in type $D_{n}$, and hence have $S_{X}=1$ or 2. In [7] it was shown that the convolution of the orbital measures of any two regular elements is absolutely continuous. The methods used there could be used to prove, more generally, that the convolution of any orbital measure with the orbital measure of a regular element is absolutely continuous. Our theorem shows that more is true.

Corollary 2. Let $X, Y$ be non-zero elements in the Lie algebra of type $B_{n}$, $C_{n}$, or $D_{n}$. If $S_{Y} \leq 2$, then $\mu_{X} * \mu_{Y}$ is absolutely continuous and $O_{X}+O_{Y}$ has non-empty interior, except if $(X, Y)$ is the exceptional pair $(S U(4), S U(2) \times S U(2))$ in $D_{4}$ where the annihilating roots of $Y$ are a subset of a Weyl conjugate of those of $X$.

Proof. This is immediate from the theorem since any non-zero $X$ has $S_{X} \leq$ $2(n-1)$ (with equality only if $X$ is type $B_{n-1}\left(C_{n-1}\right.$ or $\left.D_{n-1}\right)$ ).

Corollary 3. If $\left(X_{1}, \ldots, X_{L}\right)$ is an eligible, non-exceptional L-tuple of matrices in any of the classical Lie algebras, then there are unitarily similar matrices, $g_{i}^{-1} X_{i} g_{i}$, with the property that $\sum_{i=1}^{L} g_{i}^{-1} X_{i} g_{i}$ has distinct eigenvalues. 
Proof. This follows from the main theorem because any subset of these matrix groups with non-empty interior must contain an element with distinct eigenvalues. Indeed, the elements with distinct eigenvalues are dense.

On the other hand, if $X_{i}$ are matrices in one of the classical Lie algebras and there are unitarily similar matrices, $g_{i}^{-1} X_{i} g_{i} \in O_{X_{i}}$, with the property that $\sum_{i=1}^{L} g_{i}^{-1} X_{i} g_{i}$ has distinct eigenvalues, then $\sum O_{X_{i}}$ contains an element $Y$ with $S_{Y} \leq 2$. (Indeed, $Y$ is either type $S U(1)$ or type $B_{1}, C_{1}$ or $D_{1}$.) As noted in the first corollary, $O_{X}+O_{Y}$ has non-empty interior for any $X \neq 0$ and thus $\mu_{X_{1}} * \cdots \mu_{X_{L}} * \mu_{X}$ is absolutely continuous for any $X \neq 0$. It would be interesting to characterize the $L$-tuples for which $\sum O_{X_{i}}$ contains a matrix with distinct eigenvalues.

It is known that any $n$-fold sum of non-trivial orbits in $B_{n}, C_{n}$ or $D_{n}$ has non-empty interior. More can be said.

Corollary 4. Let $n \geq 5$. If $X_{i}$ are non-zero elements in $B_{n}\left(C_{n}\right.$ or $\left.D_{n}\right)$ for $i=1, \ldots, n-1$, then $O_{X_{1}}+\cdots+O_{X_{n-1}}$ has empty interior if and only if all $X_{i}$ are type $B_{n-1}\left(C_{n-1}\right.$ or $\left.D_{n-1}\right)$.

Proof. Suppose some $X_{i}$, say $X_{n-1}$, is not type $B_{n-1}$. Then $S_{X_{n-1}} \leq 2(n-2)$. As all $S_{X_{i}} \leq 2(n-1)$,

$$
\sum_{i=1}^{n-1} S_{X_{i}} \leq 2(n-1)(n-2)+2(n-2) \leq 2 n(n-2) .
$$

Thus $\left(X_{1}, \ldots, X_{n-1}\right)$ is eligible and non-exceptional and hence the sum of the orbits has non-empty interior. Since all root subsystems of type $B_{n-1}$ are Weyl conjugate, the converse follows from the fact that if $X$ is type $B_{n-1}$, then $\mu_{X}^{n-1}$ is not absolutely continuous 9 .

We leave it as an exercise for the reader to determine the choice of $n-1$ tuples that are not absolutely continuous when $n \leq 4$ and in type $A_{n}$.

We note that for type $A_{n}, B_{n}$ and $C_{n}$ our proof required the use of 9 only to start the induction process. In the proof given in $\mathbf{9}$ an induction argument was also used and the base cases were simply done directly. That approach could have been taken here, as well. For type $D_{n}$ our proof also used [9] to establish that when $X$ was type $S U(n)$, then $\mu_{X}^{3}$ was absolutely continuous for $n \geq 5$ and $\mu_{X}^{4}$ was absolutely continuous when $n=4$. In fact, the argument that was given there for these special types actually showed that Theorem 2 was satisfied. Thus, our theorem gives another way to deduce the formulas of $\mathbf{9}$. For example, we have

Corollary 5. Suppose $\mathfrak{g}$ is type $B_{n}$ and $X$ is type $B_{J} \times S U\left(s_{1}\right) \times \cdots \times S U\left(s_{m}\right)$.

(i) If $X$ is dominant $B$ type, then $\mu_{X}^{L}$ is absolutely continuous (and $(L) O_{X}$ has non-empty interior) if and only if $L \geq n /(n-J)$.

(ii) If $X$ is dominant $S U$ type, then $\mu_{X}^{2}$ is absolutely continuous.

Similar statements can be made for the other types, taking into account the exceptional cases.

REMARK 5. (i) We have not been able to determine if the pair of type $(S U(n)$, $S U(n-1))$ in $D_{n}$ for $n \geq 6$ is absolutely continuous. Computer results suggest that it is not for at least $n=6,7$. We remark that Prop. 3 shows that if such a pair is absolutely continuous for any $n$, say $n=n_{0}$ then, being an eligible pair, it is absolutely continuous for all $n>n_{0}$. 
(ii) It remains open to solve the analogous problem in the exceptional Lie algebras, those of type $G_{2}, F_{4}, E_{6}, E_{7}$, or $E_{8}$. In 10 the minimal $k(X)$ so that $\mu_{X}^{k(X)}$ is absolutely continuous was determined for each $X$ in the compact exceptional Lie algebras. Although the abstract root theory machinery can be applied in this setting, there is no underlying classical matrix algebra from which to derive the necessary conditions.

7.2. Orbital measures on Conjugacy classes in Compact Lie Groups. A related, but more challenging problem, is to determine which $L$-tuples, $\left(x_{1}, \ldots, x_{L}\right) \in G^{L}$, have the property that $\mu_{x_{1}} * \cdots * \mu_{x_{L}}$ is absolutely continuous with respect to Haar measure on the group $G$, when $\mu_{x}$ is the probability measure, invariant under the conjugation action of $G$ on itself, and supported on the conjugacy class generated by $x, C_{x}=\left\{g^{-1} x g: g \in G\right\}$. In [8, Thm. 9.1], the minimum integer $k(x)$ for which $\mu_{x}^{k(x)}$ is absolutely continuous was determined for all the classical Lie groups. The number $k(x)$ depended on the type of the set of annihilating roots of $x$, where in this setting by the set of annihilating roots we mean

$$
\Phi_{x}:=\{\alpha \in \Phi: \alpha(x) \equiv 0 \bmod 2 \pi\} .
$$

Again, by the type of $x$, we will mean the type of $\Phi_{x}$.

This was extended by Wright 23 to convolution products of (possibly) different $\mu_{x}$ in the case of $S U(n)$, obtaining the same characterization as for the Lie algebra problem. In this subsection, we will obtain a similar result for all the classical Lie groups whenever the group elements $x_{i}=\exp X_{i}$ where $X_{i} \in \mathfrak{g}$ and $x_{i} \in G$ have the same type.

We need the following preliminary result, analogous to Prop. 1. Given $x \in G$, we let

$$
\mathcal{N}_{x}:=\left\{R E_{\alpha}, I E_{\alpha}: \alpha(x) \neq 0 \bmod 2 \pi\right\} .
$$

LEMMA 8. (c.f. 18, 23) The measure $\mu_{x_{1}} * \cdots * \mu_{x_{L}}$ on $G_{n}$ is absolutely continuous with respect to Haar measure on $G_{n}$ if and only if any of the following hold:

(i) The set $\prod_{i=1}^{L} C_{x_{i}} \subseteq G_{n}$ has non-empty interior;

(ii) The set $\prod_{i=1}^{L} C_{x_{i}} \subseteq G_{n}$ has positive measure;

(iii) There exists $g_{i} \in G_{n}$ with $g_{1}=I d$, such that

$$
\operatorname{sp}\left\{A d\left(g_{i}\right) \mathcal{N}_{x_{i}}: i=1, \ldots, L\right\}=\mathfrak{g}_{n} .
$$

Proposition 4. Let $x_{1}, \ldots, x_{L} \in G_{n}$ and assume $x_{i}=\exp X_{i}$ for some $X_{i} \in$ $\mathfrak{g}_{n}$ where $x_{i}$ and $X_{i}$ have the same type. Then $\mu_{x_{1}} * \cdots * \mu_{x_{L}}$ is absolutely continuous with respect to Haar measure on $G_{n}$ if and only if $\mu_{X_{1}} * \cdots * \mu_{X_{L}}$ is absolutely continuous with respect to Lebesgue measure on $\mathfrak{g}_{n}$. Moreover, $\prod_{i=1}^{L} C_{x_{i}}$ has nonempty interior in $G_{n}$ if and only if $\sum_{i=1}^{L} O_{X_{i}}$ has non-empty interior in $\mathfrak{g}_{n}$.

Proof. If $x_{i}$ and $X_{i}$ are of the same type, then $\mathcal{N}_{x_{i}}=\mathcal{N}_{X_{i}}$. Consequently,

$$
\operatorname{sp}\left\{A d\left(g_{i}\right) \mathcal{N}_{x_{i}}: i=1, \ldots, L\right\}=\operatorname{sp}\left\{A d\left(g_{i}\right) \mathcal{N}_{X_{i}}: i=1, \ldots, L\right\}
$$


and thus $\mu_{x_{1}} * \cdots * \mu_{x_{L}}$ is absolutely continuous if and only if $\mu_{X_{1}} * \cdots * \mu_{X_{L}}$ is absolutely continuous. The latter statement holds as absolute continuity is equivalent to non-empty interior of either the product of conjugacy classes or the sum of orbits, depending on the setting.

REMARK 6. If $x_{i}=\exp X_{i}$ and $\mu_{X_{1}} * \cdots * \mu_{X_{L}}$ is not absolutely continuous, then it still follows $\mu_{x_{1}} * \cdots * \mu_{x_{L}}$ is not absolutely continuous. We simply note that always $\Phi_{X_{i}} \subseteq \Phi_{x_{i}}$.

Consider the Lie group $S U(n)$. Every conjugacy class contains a diagonal matrix so in studying the measure $\mu_{x}$ there is no loss in assuming

$$
x=\operatorname{diag}\left(\exp i a_{1}, \ldots, \exp i a_{n}\right)
$$

where $a_{j} \in[0,2 \pi)$ and $\sum a_{j} \equiv 0 \bmod 2 \pi$. Notice that $x=\exp X$ where $X=$ $\operatorname{diag}\left(i a_{1}, \ldots, i a_{n}\right)$ belongs to $s u(n)$. The root $\alpha=e_{j}-e_{k}$ acts on $x$ (and $X$ ) by $\alpha(x)=a_{j}-a_{k}$. Thus $\Phi_{x}=\Phi_{X}$ and so the Proposition applies to all $L$-tuples in $S U(n)$.

This is not true for the other classical Lie groups. For example, in $S O(2 n+1)$ (type $B_{n}$ ) there is an element $x$ with $\Phi_{x}=\left\{e_{i} \pm e_{j}: 1 \leq i \neq j \leq n\right\}$, i.e., of type $D_{n}$. This type does not arise in the Lie algebra. Indeed, the only element $X \in \operatorname{so}(2 n+1)$ with $\Phi_{X} \supseteq \Phi_{x}$ is $X=0$. The element $x$ has the property that $\mu_{x}^{2 n} \in L^{1}(G)$, but $\mu_{x}^{2 n-1}$ is singular with respect to Haar measure on $G$. In contrast, any $X$ with $\exp X=x$ has $\mu_{X}^{2} \in L^{2}(\mathfrak{g})$.

These additional (and often more complicated) types of elements that can arise in the Lie groups make the problem of characterizing absolute continuity of orbital measures on Lie groups more challenging than for Lie algebras.

\section{References}

[1] A. Dooley, J. Repka and N. Wildberger, Sums of adjoint orbits, Linear and multilinear algebra 36(1993), 79-101.

[2] A. Frumkin and A. Goldberger, On the distribution of the spectrum of the sum of two Hermitian or real symmetric spaces, Adv. Appl. Math. 37(2006), 268-286.

[3] P. Gratczyk and P. Sawyer, On the kernel of the product formula on symmetric spaces, J. Geom. Anal. 14(2004), 653-672.

[4] P. Gratczyk and P. Sawyer, Absolute continuity of convolutions of orbital measures on Riemannian symmetric spaces, J. Func. Anal. 259(2010), 1759-1770.

[5] P. Gratczyk and P. Sawyer, A sharp criterion for the existence of the density in the product formula on symmetric spaces of Type $A_{n}$, J. Lie Theory 20(2010), 751-766.

[6] S.K. Gupta and K.E. Hare, Singularity of orbits in classical Lie algebras, Geom. Func. Anal. 13(2003), 815-844.

[7] S.K. Gupta and K.E. Hare, Convolutions of generic orbital measures in compact symmetric spaces, Bull. Aust. Math. Soc. 79(2009), 513-522.

[8] S.K. Gupta and K.E. Hare, $L^{2}$-singular dichotomy for orbital measures of classical compact Lie groups, Adv. Math. 222(2009), 1521-1573.

[9] S.K. Gupta, K.E. Hare and S. Seyfaddini, $L^{2}$-singular dichotomy for orbital measures of classical simple Lie algebras, Math. Zeit. 262(2009), 91-124.

[10] K.E. Hare, D. Johnstone, F. Shi and M. Yeung, $L^{2}$-singular dichotomy for exceptional Lie groups and algebras, J. Aust. Math. Soc. 95(2013), 362-382.

[11] S. Helgason, Differential geometry, Lie groups and symmetric spaces, Academic Press, New York, 1978.

[12] J. Humphreys, Introduction to Lie algebras and representation theory, Springer-Verlag, New York, 1972.

[13] S. Lang, Differential and Riemannian manifolds, Springer-Verlag, New York, 1995.

[14] A. Knapp, Lie groups beyond an introduction, Birkhauser, Verlag AG, 2002. 
[15] A. Knutson and T. Tao, Honeycombs and sums of Hermitian matrices, Notices Amer. Math. Soc. 48(2001), 175-186.

[16] M. Mimura and H. Toda, Topology of Lie groups, Trans. Math. Monographs 91, Trans. Amer. Math. Soc., Providence, R.I., 1991.

[17] D. Ragozin, Zonal measure algebras on isotropy irreducible homogeneous spaces, J. Func. Anal. 17(1974), 355-376.

[18] D. Ragozin, Central measures on compact simple Lie groups, J. Func. Anal. 10(1972), 212229.

[19] F. Ricci and E. Stein, Harmonic analysis on nilpotent groups and singular integrals. II. Singular kernels supported on submanifolds, J. Func. Anal. 78(1988), 56-84.

[20] F. Ricci and E. Stein, Harmonic analysis on nilpotent groups and singular integrals. III. Fractional integration along manifolds, J. Func. Anal. 86(1989), 360-389.

[21] V.S. Varadarajan, Lie groups and Lie algebras and their representations, Springer-Verlag, New York, 1984.

[22] N. Wildberger, On a relationship between adjoint orbits and conjugacy classes of a Lie group, Can. Math. Bull. 33(1990), 297-304.

[23] A. Wright, Sums of adjoint orbits and $L^{2}$-singular dichotomy for $S U(m)$, Adv. Math. $\mathbf{2 2 7}(2011)$ 253-266.

Dept. of Mathematics and Statistics, Sultan Qaboos University, P.O. Box 36 Al Khodh 123, Sultanate of Oman

E-mail address: gupta@squ.edu.om

Dept. of Pure Mathematics, University of Waterloo, Waterloo, Ont., Canada, N2L 3G1

E-mail address: kehare@uwaterloo.ca 